\title{
Doppler follow-up of OGLE transiting companions in the Galactic bulge ${ }^{\star}$
}

\author{
F. Bouchy ${ }^{1,4}$, F. Pont ${ }^{1,4}$, C. Melo ${ }^{2}$, N. C. Santos ${ }^{3,4}$, M. Mayor ${ }^{4}$, D. Queloz ${ }^{4}$, and S. Udry ${ }^{4}$ \\ ${ }^{1}$ Laboratoire d'Astrophysique de Marseille, Traverse du Siphon, BP 8, 13376 Marseille Cedex 12, France \\ e-mail: Francois.Bouchy@oamp. fr \\ 2 European Southern Observatory, Casilla 19001, Santiago 19, Chile \\ 3 Centro de Astronomia e Astrofísica da Universidade de Lisboa, Tapada da Ajuda, 1349-018 Lisboa, Portugal \\ 4 Observatoire de Genève, 51 ch. des Maillettes, 1290 Sauverny, Switzerland
}

Received 23 July 2004 / Accepted 13 October 2004

\begin{abstract}
Two years ago, the OGLE-III survey (Optical Gravitational Lensing Experiment) announced the detection of 54 short period multi-transiting objects in the Galactic bulge (Udalski et al. 2002a,b). Some of these objects were considered to be potential hot Jupiters. In order to determine the true nature of these objects and to characterize their actual mass, we conducted a radial velocity follow-up of 18 of the smallest transiting candidates. We describe here our procedure and report the characterization of 8 low-mass star-transiting companions, 2 grazing eclipsing binaries, 2 triple systems, 1 confirmed exoplanet (OGLE-TR-56b), 1 possible exoplanet (OGLE-TR-10b), 1 clear false positive and 3 unsolved cases. The variety of cases encountered in our follow-up covers a large part of the possible scenarios occurring in the search for planetary transits. As a by-product our program yields precise masses and radii of low mass stars.
\end{abstract}

Key words. techniques: radial velocities - stars: binaries: eclipsing - stars: low-mass, brown dwarfs - stars: planetary systems

\section{Introduction}

Since 1995 the search for planets by radial velocity surveys has led to the detection of more than 120 planetary candidates. The diversity of orbital characteristics, the mass distribution of the planets (actually only the $m \sin i$ ) and its link with brown dwarfs and low-mass stars as well as the characteristics of host stars prompted a reexamination of planetary formation theory (e.g., Udry et al. 2003; Santos et al. 2003; Eggenberger et al. 2004). The most unexpected fact was the existence of extrasolar giant planets (EGPs) in very close orbits. Additional mechanisms, not envisioned in the study of our Solar system, have been suggested to explain these objects, like the migration of planets in the proto-planetary disk and gravitational interactions (e.g., Goldreich \& Tremaine 1980; Lin et al. 1996).

Monitoring of photometric transits caused by an EGP passing before the disk of its hosting star and obscuring part of its surface provides the opportunity to determine its actual size. When combined with spectroscopic observations, it leads to the unambiguous characterization of the two fundamental parameters (mass and radius) used for internal structure studies of EGPs. The discovery of HD 209458 by both Doppler measurements (Mazeh et al. 2000) and photometric transit

^ Based on observations collected with the UVES and FLAMES spectrographs at the VLT/UT2 Kueyen telescope (Paranal Observatory, ESO, Chile: program 70.C-0209 and 71.C-0251).
(Charbonneau et al. 2000; Henry et al. 2000) led to the first complete characterization of an EGP, illustrating the real complementarity of the two methods. These last years many extensive ground-based photometric programs have been initiated to detect transits by short period EGPs (Horne 2003). The OGLE-III survey (Optical Gravitational Lensing Experiment) recently announced the detection of 137 short-period multitransiting objects (Udalski et al. 2002a,b,c, 2003). The estimated radii of these objects range from 0.5 Jupiter radius to 0.5 solar radius and their orbital periods range from 0.8 to 8 days. The smallest objects could be suspected to be EGPs, but considering only the radius measured by OGLE one can not conclude on the planetary nature of the objects per se. They could as well be brown dwarfs or low-mass stars since in the low mass regime the radius is independent of the mass (Guillot 1999). No information on the mass of these companions is given by the transit measurements. Doppler follow-up of these candidates is the only way to confirm the planetary, brown dwarf or low-mass-star nature of the companions. Planetary transit detection suffers also some ambiguity related to the configuration of the system. The radial velocity measurement is therefore very important to discriminate true central transits from other cases such as, for example, grazing eclipsing binaries, blended systems and stellar activity. The spectroscopy of the central star, which is a by-product of the radial velocity measurement, is necessary to constrain the radius of the 
star and thence of the companion. The measurement of the true mass of a companion by the radial velocity orbit, coupled with the measurement of its radius, leads to a direct measurement of its mean density, an essential parameter for the study of the internal structure of EGPs, brown dwarfs and low-mass stars.

The difficulties of Doppler follow-up of OGLE candidates come from the faintness of the stars (with $V$ magnitudes in the range 14-18). Furthermore, the fields, which are located in the Galactic disk, are very crowed. To characterize a hot Jupiter, one needs radial velocity precision better than $100 \mathrm{~m} \mathrm{~s}^{-1}$ and the capability to distinguish whether the system is blended by a third star like the triple system HD 41004 (Santos et al. 2003).

Several teams are involved in the Doppler follow-up of OGLE candidates. Konacki et al. (2003a) announced first that the companion of OGLE-TR-56 is a planet of 0.9 Jupiter mass. Dreizler et al. (2003) gave an upper limit of 2.5 Jupiter mass for the companion of OGLE-TR-3. This object was however refuted by Konacki et al. (2003b) who also gave information on 3 other OGLE companions (OGLE-TR-10, 33 and 58). Additional measurements, conducted by Torres et al. (2004a), lead to improving the mass determination of OGLE-TR-56b to 1.45 Jupiter mass. Recently, we announced the characterization of planets OGLE-TR-113b, OGLE-TR-132b (Bouchy et al. 2004, Moutou et al. 2004) and OGLE-TR-111b (Pont et al. 2004).

We present in this paper the Doppler follow-up observations of 18 OGLE multi-transiting companions (OGLE-TR-5, $6,7,8,10,12,17,18,19,33,34,35,48,49,55,56,58$ and 59) from the 54 detected in the Galactic bulge (Udalski et al. 2002a,b).

\section{Target selection and observations}

\subsection{UVES}

We obtained $16 \mathrm{~h}$ in service mode with the UVES spectrograph on the ESO-VLT in October 2002 (program 70C.0209A). For this run we selected 3 candidates (OGLE-TR-8, 10 and 12) with an estimated companion radius less than 1.6 Jupiter radius (following the value given by Udalski et al. 2002a). We were aware that UVES has no fiber to produce a stable illumination at the entrance of the spectrograph and that the Iodine cell method is unusable for such faint stars. However, using the smallest slit $(0.3 \mathrm{arcsec})$ in medium seeing conditions $(0.9-1.4 \mathrm{arcsec})$, we can minimize the velocity error stemming from the shift of the photo-center on the slit. Considering as well the very good guiding $(<0.1$ arcsec) and the centering accuracy of UT2 (about $0.1 \mathrm{arcsec}$ ), we estimated that for a 1.0 arcsec seeing one should reach an overall stability of the photo-center of about 1/70 of the slit width. This corresponds to a radial velocity error of about $40 \mathrm{~m} \mathrm{~s}^{-1}$. In order to check our accuracy we added the bright standard radial velocity star HD 162907 (selected from the CORALIE exoplanet survey) close to our 3 candidates. Moreover, in order to track and to correct instrumental calibration drifts we took thorium exposures before and after each science exposure. With such a procedure we expected an overall radial velocity precision of about $50 \mathrm{~m} \mathrm{~s}^{-1}$. We used the red arm of the spectrograph with a central wavelength of $580 \mathrm{~nm}$.

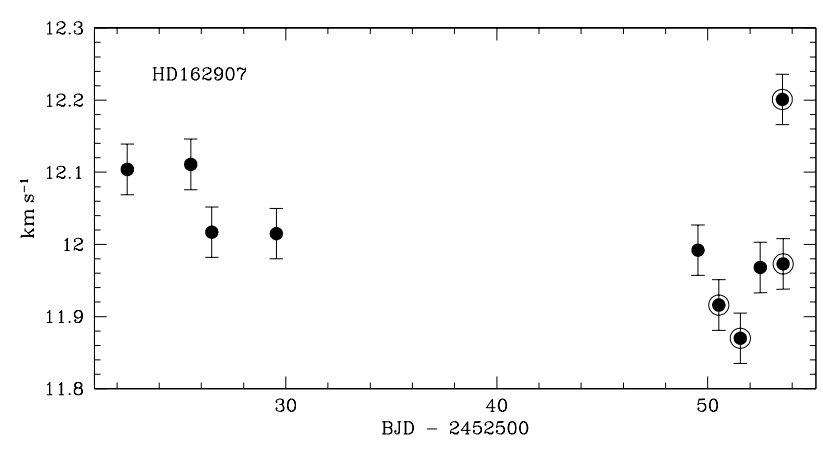

Fig. 1. Doppler measurements of the standard star HD 162907 made with UVES. The dispersion of $93 \mathrm{~m} \mathrm{~s}^{-1}$ is dominated by the centering error. Encircled points correspond to measurements made with a seeing lower than 0.9 arcsec.

Notice that in order to reach this precision it is mandatory not to conduct this program in good seeing conditions (i.e. seeing less than 0.9 arcsec). We noticed that such an unusual requirement is extremely rare in Paranal. We made 8 measurements of each target with exposure times between 20 and $40 \mathrm{~min}$. Figure 1 shows the result obtained with UVES on HD 162907. The dispersion of $93 \mathrm{~m} \mathrm{~s}^{-1}$ is dominated by the centering error, especially during nights with good seeing conditions. If we eliminate the 4 measurements made with a seeing lower than 0.9 arcsec, the dispersion reaches $54 \mathrm{~m} \mathrm{~s}^{-1}$.

\subsection{FLAMES}

The FLAMES facility on the VLT (available since march 2003) seems to be the most efficient way to conduct the Doppler follow-up of OGLE candidates. FLAMES is a multi-fiber link which makes it possible to feed the spectrograph UVES with up to 7 targets in a field-of-view of 25 arcmin diameter in addition to the simultaneous thorium calibration. The fiber link allows a stable illumination at the entrance of the spectrograph and the simultaneous thorium calibration is used to track instrumental drift. In $45 \mathrm{~min}$ on a $17 \mathrm{mag}$ star a signal-to-noise ratio is reached of about 8 , corresponding to a photon noise uncertainty of about $25 \mathrm{~m} \mathrm{~s}^{-1}$ on a non-rotating $\mathrm{K}$ dwarf star. We obtained $24 \mathrm{~h}$ in service mode on this instrument (program 71.C-0251A) in order to observe in May and June 200317 candidates (OGLE-TR-5, 6, 7, 10, 12, 17, 18, 19, 33, 34, 35, 48, 49, 55, 56, 58 and 59) located in 3 FLAMES fields (see Fig. 2). For this program, we selected a sample including 7 candidates with an estimated companion radius less than 1.6 Jupiter radius (following the value given by Udalski et al. 2002a,b) and complemented each field with targets having larger companion radius in order to constrain the mass-radius relation of low-mass stars.

\subsection{HARPS}

HARPS is a new fiber-fed spectrograph on the ESO 3.6-m telescope (Mayor et al. 2003) dedicated to high-precision Doppler measurements. During the second commissioning in June 2003 we tested the capability of the instrument to realize radial velocity measurements on faint stars and made 5 measurements 


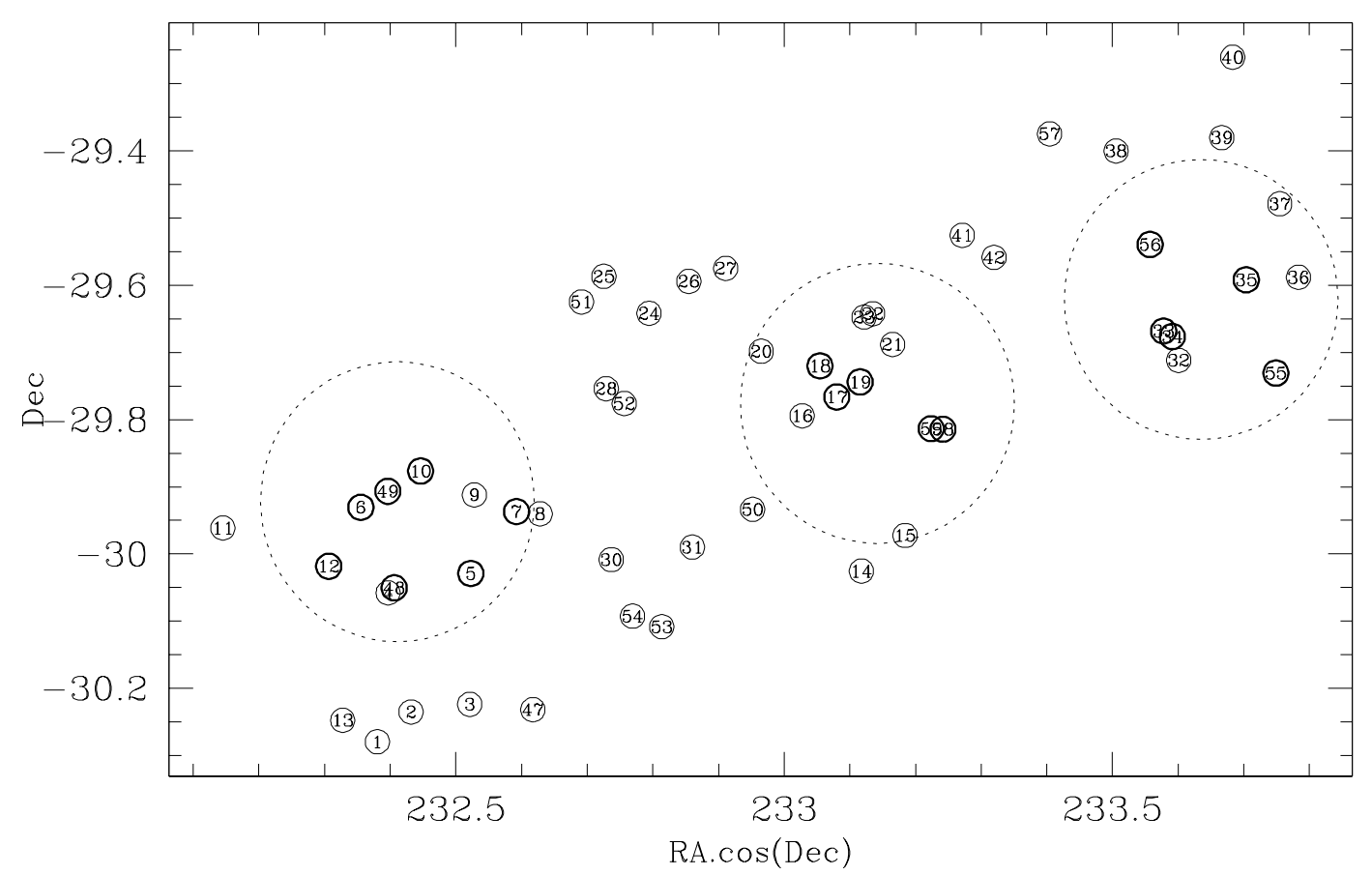

Fig. 2. Positions of the 54 OGLE candidates on the sky and location of our 3 selected FLAMES fields. Bold circles correspond to the 17 OGLE candidates observed during our run.

of 1-hour exposure on OGLE-TR-56. The first 20 blue spectral orders were not used to compute radial velocity because of their too low $S / N(<1)$.

\section{Spectroscopic analysis}

\subsection{Radial velocities}

The spectra obtained from the FLAMES and UVES spectrographs were extracted using the standard ESO-pipeline with bias, flat-field and background correction. Wavelength calibration was performed with ThAr spectra. The radial velocities were obtained by weighted cross-correlation with a numerical mask constructed from the Solar spectrum atlas. In the case of FLAMES and HARPS, the simultaneous ThAr spectrum was used to compute the instrumental drift by cross-correlation with a thorium mask. Radial velocity uncertainties (in $\mathrm{km} \mathrm{s}^{-1}$ ) were computed as a function of the SNR per pixel of the spectrum, the width $\left(F W H M\right.$ in $\left.\mathrm{km} \mathrm{s}^{-1}\right)$ and depth $(C$ in $\%)$ of the Cross-Correlation Function $(\mathrm{CCF})$ through the following relation based on photon noise simulations:

$\sigma_{\mathrm{RV}}=\frac{3 \cdot \sqrt{F W H M}}{S N R \cdot C}$.

However, our measurements are clearly not photon-noiselimited and we added quadratically an empirically determined uncertainty of $35 \mathrm{~m} \mathrm{~s}^{-1}$ in order to take into account the systematic errors probably due to wavelength calibration errors, fiber-to-fiber contamination, and residual cosmic rays. We checked that, for non-rotating dwarf stars, the $\mathrm{O}-\mathrm{C}$ residuals of our measurements are in agreement with the estimated uncertainties based on this relation. Our best $\mathrm{O}-\mathrm{C}$ residuals indicate that we reached a radial velocity precision lower than $100 \mathrm{~m} \mathrm{~s}^{-1}$.
Our radial velocity measurements and Cross-Correlation Function parameters are listed in Table 1. For some candidates observed with FLAMES at very low SNR $(S N R<5)$, the depth of the CCF is correlated with the SNR. This is a clear indication that the spectra are contaminated by background light due in part to the fiber-to-fiber contamination. We took this effect into consideration in order to correctly estimate the SNR and to compute our radial velocity uncertainties more strictly. Phasefolded radial velocities and results are presented and discussed in Sect. 5.

\subsection{Rotation velocities}

For each object the eight observed cross-correlation function were shifted by the observed radial velocity and coadded to give a combined CCF of higher signal-to-noise ratio. Rotationally broadened line profiles were convolved with a Gaussian instrumental profile depending of the instrument and correlation mask: $\sigma=3.0 \mathrm{~km} \mathrm{~s}^{-1}$ for UVES and $4.0 \mathrm{~km} \mathrm{~s}^{-1}$ for FLAMES. The instrumental profile was determined with HD 162907 for UVES and the combined spectrum of OGLETR-19 and OGLE-TR-49 for FLAMES. We also checked the instrumental profile on both spectrographs with the ThAr spectrum. The profiles were fitted to the CCF to determine the projected rotation velocity $v \sin i$ of the target objects. The result is displayed in Table 2. A quadratic limb-darkening with coefficients $u 1+u 2=0.6$ was assumed. The computations of Barban et al. (2003) find that such a coefficient is a suitable approximation for a wide range of spectral types in wavelengths corresponding to the $V$ filter.

For close binaries, with rotation periods of the order of a few days, we expect that the rotation axis is aligned with the 
Table 1. Radial velocity measurements (in the barycentric frame) and CCF parameters. Labels $a$ and $b$ indicate that 2 components are present in the CCF. BJD in the range [522-557], [750-798] and [809-812] correspond respectively to UVES, FLAMES and HARPS measurements.

\begin{tabular}{rrrrrr}
\hline \hline $\begin{array}{r}\text { BJD } \\
{[-2452000 \mathrm{~d}]}\end{array}$ & $\begin{array}{r}R V \\
{\left[\mathrm{~km} \mathrm{~s}^{-1}\right]}\end{array}$ & $\begin{array}{r}\text { depth } \\
{[\%]}\end{array}$ & $\begin{array}{r}F W H M \\
{\left[\mathrm{~km} \mathrm{~s}^{-1}\right]}\end{array}$ & $S N R$ & $\begin{array}{r}\sigma_{\mathrm{RV}} \\
{\left[\mathrm{km} \mathrm{s}^{-1}\right]}\end{array}$ \\
\hline OGLE-5 & & & & & \\
\hline 759.72111 & -15.452 & 1.88 & 119.4 & 8.9 & 1.959 \\
764.85708 & 13.273 & 2.17 & 151.5 & 7.6 & 2.239 \\
766.79458 & 48.733 & 2.28 & 116.7 & 11.6 & 1.226 \\
767.72839 & 12.256 & 1.83 & 142.5 & 8.8 & 2.224 \\
768.84710 & -11.662 & 2.56 & 119.0 & 15.5 & 0.825 \\
769.85598 & 55.993 & 2.33 & 121.4 & 10.0 & 1.419 \\
770.82050 & 60.894 & 2.34 & 122.9 & 11.0 & 1.293 \\
797.76880 & -34.035 & 2.40 & 123.3 & 11.3 & 1.229 \\
\hline OGLE-6 & & & & & \\
\hline 759.72111 & 33.160 & 7.34 & 35.4 & 5.0 & 0.488 \\
764.85708 & 46.860 & 9.95 & 34.9 & 6.4 & 0.281 \\
766.79458 & -10.166 & 10.93 & 34.0 & 7.1 & 0.228 \\
767.72839 & -10.385 & 8.99 & 33.2 & 5.6 & 0.345 \\
768.84710 & 33.995 & 13.55 & 38.0 & 10.5 & 0.135 \\
769.85598 & 44.341 & 10.22 & 36.2 & 6.1 & 0.292 \\
770.82050 & 8.344 & 12.33 & 35.0 & 7.2 & 0.203 \\
797.76880 & 24.197 & 11.86 & 36.6 & 7.5 & 0.207 \\
\hline OGLE-7 & & & & & \\
\hline 759.72111 & 8.454 & 6.94 & 55.3 & 9.6 & 0.337 \\
764.85708 & 20.473 & 7.13 & 54.6 & 10.4 & 0.301 \\
766.79458 & -11.924 & 8.32 & 54.8 & 11.3 & 0.239 \\
767.72839 & 14.997 & 6.79 & 48.6 & 9.5 & 0.326 \\
768.84710 & -42.394 & 8.04 & 54.7 & 14.4 & 0.195 \\
769.85598 & 10.541 & 7.13 & 50.4 & 10.9 & 0.276 \\
770.82050 & -6.034 & 7.48 & 50.6 & 11.2 & 0.257 \\
797.76880 & 9.175 & 7.30 & 52.4 & 12.0 & 0.250 \\
\hline OGLE-8a & & & & & \\
\hline 522.55485 & 19.193 & 10.94 & 16.9 & 6.9 & 0.167 \\
526.55409 & 69.882 & 11.60 & 18.1 & 8.6 & 0.133 \\
549.59728 & 29.917 & 10.50 & 16.8 & 4.1 & 0.288 \\
550.58081 & -54.831 & 11.04 & 17.3 & 5.1 & 0.224 \\
551.59645 & -78.950 & 11.02 & 17.7 & 5.9 & 0.197 \\
552.54163 & -17.312 & 11.72 & 17.9 & 7.2 & 0.154 \\
553.59422 & 64.676 & 10.67 & 17.4 & 5.9 & 0.202 \\
556.50991 & -79.423 & 10.34 & 17.0 & 4.2 & 0.287 \\
\hline & & & & & \\
\hline & & & & \\
\hline
\end{tabular}

orbital axis, the orbit is circularized and the system is tidally locked (e.g., Levato 1976; Hut 1981; Melo et al. 2001). For known close binaries, the alignment of the axes and the tidal locking are observed to be effective even before orbital circularization. It can therefore be expected that in cases of a massive transiting companion with a short period, the system is tidally locked and $v \sin i$ is large. In that case, $P_{\text {rot }}=P_{\text {transit }}$ and the rotational velocity is directly related to the radius of the primary. Rotation velocities observed in our sample are generally compatible with the hypothesis of tidal locking. In these cases $v \sin i$ provides a measurement of $R$ with an estimated accuracy of a few percent. The uncertainty in the determination of $v \sin i$ was estimated by computing values for each of the individual CCF and calculating the dispersion of these values. In most cases this "formal" uncertainty is very small, and the
Table 1. continued.

\begin{tabular}{|c|c|c|c|c|c|}
\hline $\begin{array}{r}\text { BJD } \\
{[-2452000 \mathrm{~d}]}\end{array}$ & $\begin{array}{r}R V \\
{\left[\mathrm{~km} \mathrm{~s}^{-1}\right]}\end{array}$ & $\begin{array}{r}\text { depth } \\
{[\%]}\end{array}$ & $\begin{array}{r}F W H M \\
{\left[\mathrm{~km} \mathrm{~s}^{-1}\right]}\end{array}$ & $\overline{S N R}$ & $\begin{array}{r}\sigma_{\mathrm{RV}} \\
{\left[\mathrm{km} \mathrm{s}^{-1}\right]}\end{array}$ \\
\hline \multicolumn{6}{|l|}{ OGLE-8b } \\
\hline 522.55485 & -27.289 & 10.33 & 16.1 & 6.9 & 0.172 \\
\hline 526.55409 & -80.238 & 10.35 & 16.7 & 8.6 & 0.142 \\
\hline 549.59728 & -37.875 & 9.54 & 17.3 & 4.1 & 0.321 \\
\hline 550.58081 & 48.938 & 9.70 & 17.1 & 5.1 & 0.253 \\
\hline 551.59645 & 74.409 & 10.02 & 17.7 & 5.9 & 0.216 \\
\hline 552.54163 & 10.650 & 9.87 & 17.2 & 7.2 & 0.179 \\
\hline 553.59422 & -74.939 & 10.29 & 15.4 & 5.9 & 0.197 \\
\hline 556.50991 & 74.659 & 9.14 & 15.4 & 4.2 & 0.309 \\
\hline \multicolumn{6}{|l|}{ OGLE-10 } \\
\hline 522.50823 & -6.421 & 30.62 & 11.7 & 6.1 & 0.065 \\
\hline 525.50905 & -6.413 & 30.14 & 11.9 & 6.8 & 0.061 \\
\hline 526.51146 & -6.485 & 29.06 & 10.7 & 6.9 & 0.060 \\
\hline 549.55329 & -6.607 & 29.94 & 11.9 & 4.4 & 0.086 \\
\hline 550.53700 & -6.135 & 29.86 & 11.5 & 3.4 & 0.106 \\
\hline 551.55409 & -6.475 & 30.28 & 11.2 & 3.8 & 0.094 \\
\hline 552.50287 & -6.410 & 31.03 & 11.9 & 6.4 & 0.063 \\
\hline 553.54729 & -6.135 & 30.58 & 11.3 & 6.4 & 0.062 \\
\hline 759.72111 & -6.291 & 24.03 & 12.9 & 8.4 & 0.064 \\
\hline 764.85708 & -6.236 & 27.18 & 13.1 & 10.9 & 0.051 \\
\hline 766.79458 & -6.212 & 26.17 & 13.1 & 9.8 & 0.055 \\
\hline 767.72839 & -6.274 & 25.11 & 12.5 & 9.0 & 0.059 \\
\hline 768.84710 & -6.365 & 27.19 & 12.8 & 14.6 & 0.044 \\
\hline 769.85598 & -6.238 & 25.91 & 12.9 & 9.9 & 0.055 \\
\hline 770.82050 & -6.105 & 26.89 & 12.5 & 15.3 & 0.043 \\
\hline 797.76880 & -6.130 & 26.80 & 12.6 & 11.2 & 0.050 \\
\hline \multicolumn{6}{|l|}{ OGLE-12 } \\
\hline 522.52429 & -10.897 & 27.06 & 11.8 & 8.0 & 0.059 \\
\hline 525.52490 & 43.661 & 27.50 & 11.7 & 8.1 & 0.058 \\
\hline 526.52813 & 57.877 & 27.47 & 12.0 & 9.0 & 0.055 \\
\hline 549.57081 & -0.796 & 26.78 & 12.3 & 5.4 & 0.081 \\
\hline 550.55534 & 21.265 & 27.57 & 11.5 & 4.3 & 0.093 \\
\hline 551.57020 & 45.212 & 27.21 & 11.6 & 7.0 & 0.064 \\
\hline 552.51801 & 58.079 & 27.61 & 12.2 & 7.4 & 0.062 \\
\hline 553.56592 & 54.157 & 26.78 & 12.3 & 5.6 & 0.078 \\
\hline 759.72111 & 52.149 & 20.08 & 12.9 & 6.6 & 0.089 \\
\hline 764.85708 & -10.531 & 22.86 & 12.4 & 8.0 & 0.068 \\
\hline 766.79458 & 16.577 & 23.38 & 13.3 & 9.3 & 0.061 \\
\hline 767.72839 & 39.484 & 21.31 & 12.8 & 8.2 & 0.071 \\
\hline 768.84710 & 57.534 & 24.75 & 13.2 & 15.4 & 0.045 \\
\hline 769.85598 & 56.098 & 22.96 & 12.6 & 8.0 & 0.068 \\
\hline 770.82050 & 39.236 & 24.05 & 13.2 & 11.6 & 0.052 \\
\hline 797.76880 & 15.003 & 23.58 & 12.8 & 9.4 & 0.060 \\
\hline
\end{tabular}

dominant source of error is actually the adopted value of the limb darkening coefficient (see Sect. 6). For the smallest rotational velocities $\left(v \sin i<5 \mathrm{~km} \mathrm{~s}^{-1}\right)$, the dominant uncertainty becomes the adopted value of the instrumental broadening and the stellar micro-turbulence parameter. In order to take such systematic uncertainties into account we afterward fix the lowest uncertainty of $v \sin i$ to $1 \mathrm{~km} \mathrm{~s}^{-1}$.

\subsection{Stellar spectroscopic parameters}

For the slowly-rotating stars in our sample the stellar parameters (temperatures, gravities and metallicities) were obtained from an analysis of a set of Fe I and Fe II lines, following the 
Table 1. continued.

\begin{tabular}{rrrrrr}
\hline \hline BJD & $\begin{array}{r}R V \\
{[-2452000 \mathrm{~d}]}\end{array}$ & $\begin{array}{r}\text { depth } \\
{\left[\mathrm{km} \mathrm{s}^{-1}\right]}\end{array}$ & $\begin{array}{r}F W H M \\
{\left[\mathrm{~km} \mathrm{~s}^{-1}\right]}\end{array}$ & $S N R$ & $\begin{array}{r}\sigma_{\mathrm{RV}} \\
{\left[\mathrm{km} \mathrm{s}^{-1}\right]}\end{array}$ \\
\hline OGLE-17 & & & & & \\
\hline 759.75994 & -64.941 & 26.73 & 10.0 & 7.7 & 0.058 \\
764.81842 & -9.416 & 26.36 & 10.3 & 6.1 & 0.069 \\
766.84092 & -2.377 & 26.49 & 9.8 & 6.9 & 0.062 \\
767.76871 & -8.671 & 17.55 & 9.3 & 3.8 & 0.142 \\
768.76694 & -19.328 & 28.28 & 10.0 & 8.5 & 0.053 \\
769.81610 & -32.710 & 26.32 & 10.1 & 7.5 & 0.060 \\
770.90035 & -46.353 & 24.91 & 9.7 & 6.5 & 0.067 \\
791.72546 & -21.462 & 26.47 & 9.9 & 7.5 & 0.059 \\
\hline OGLE-18 & & & & & \\
\hline 759.75994 & -24.248 & 4.67 & 87.5 & 8.4 & 0.716 \\
764.81842 & -12.247 & 4.13 & 72.2 & 7.1 & 0.870 \\
766.84092 & 0.683 & 4.26 & 66.8 & 7.3 & 0.789 \\
767.76871 & -91.001 & 3.75 & 60.9 & 5.0 & 1.249 \\
768.76694 & -8.342 & 4.55 & 78.8 & 8.9 & 0.659 \\
769.81610 & -73.094 & 4.90 & 69.6 & 8.2 & 0.624 \\
770.90035 & -23.657 & 4.29 & 82.5 & 7.1 & 0.895 \\
791.72546 & -29.868 & 4.23 & 81.9 & 8.4 & 0.765 \\
\hline OGLE-19 & & & & & \\
\hline 759.75994 & -33.380 & 25.46 & 10.0 & 5.3 & 0.078 \\
764.81842 & -33.245 & 24.85 & 9.5 & 4.9 & 0.084 \\
766.84092 & -33.287 & 25.14 & 9.9 & 4.8 & 0.086 \\
767.76871 & -33.385 & 17.80 & 9.3 & 3.6 & 0.148 \\
768.76694 & -33.087 & 29.12 & 9.9 & 5.9 & 0.065 \\
769.81610 & -33.244 & 25.75 & 9.8 & 5.2 & 0.078 \\
770.90035 & -33.394 & 23.78 & 10.2 & 4.6 & 0.095 \\
791.72546 & -33.414 & 24.53 & 9.6 & 5.2 & 0.080 \\
\hline OGLE-33a & & & & & \\
\hline 764.89532 & -28.500 & 4.00 & 61.2 & 17.2 & 0.343 \\
766.87982 & -28.500 & 4.00 & 61.2 & 16.0 & 0.368 \\
767.80618 & -28.500 & 4.00 & 61.2 & 15.6 & 0.378 \\
769.77114 & -28.500 & 4.00 & 61.2 & 16.9 & 0.349 \\
770.85930 & -28.500 & 4.00 & 61.2 & 16.1 & 0.366 \\
790.70926 & -28.500 & 4.00 & 61.2 & 16.8 & 0.351 \\
797.73001 & -28.500 & 4.00 & 61.2 & 17.1 & 0.345 \\
\hline OGLE-33b & & & & & \\
\hline 764.89532 & 1.895 & 0.70 & 52.0 & 17.2 & 1.797 \\
766.87982 & 9.212 & 0.60 & 51.0 & 16.0 & 2.232 \\
767.80618 & -63.941 & 1.00 & 48.0 & 15.6 & 1.333 \\
769.77114 & -62.742 & 1.00 & 53.0 & 16.9 & 1.293 \\
770.85930 & 26.163 & 0.40 & 49.0 & 16.1 & 3.261 \\
790.70926 & 22.695 & 0.50 & 52.0 & 16.8 & 2.576 \\
\hline 797.73001 & -60.852 & 1.10 & 49.0 & 17.1 & 1.117 \\
\hline & & & & &
\end{tabular}

procedure used in Santos et al. (2004). Line equivalent widths were derived using IRAF ${ }^{1}$, and the abundances were obtained using a revised version of the code MOOG (Sneden 1973), and a grid of Kurucz (1993) atmospheres.

The final parameters have errors of the order of $200 \mathrm{~K}$ in $T_{\text {eff }}, 0.40$ in $\log g$, and $0.20 \mathrm{dex}$ in $[\mathrm{Fe} / \mathrm{H}]$ (see Table 2). The precision of the derived atmospheric parameters is most affected by relatively low $\mathrm{S} / \mathrm{N}$ of the combined spectra (30-50), together with some possible contamination coming from the ThAr spectrum. Furthermore, blends with the spectrum of a

${ }^{1}$ Distributed by NOAO, AURA, Inc., under contract with the NSF,
Table 1. continued.

\begin{tabular}{|c|c|c|c|c|c|}
\hline $\begin{array}{r}\text { BJD } \\
{[-2452000 \mathrm{~d}]}\end{array}$ & $\begin{array}{r}R V \\
{\left[\mathrm{~km} \mathrm{~s}^{-1}\right]}\end{array}$ & $\begin{array}{r}\text { depth } \\
{[\%]}\end{array}$ & $\begin{array}{r}F W H M \\
{\left[\mathrm{~km} \mathrm{~s}^{-1}\right]}\end{array}$ & SNR & $\begin{array}{r}\sigma_{\mathrm{RV}} \\
{\left[\mathrm{km} \mathrm{s}^{-1}\right]}\end{array}$ \\
\hline \multicolumn{6}{|l|}{ OGLE-34 } \\
\hline 759.79751 & 32.726 & 21.74 & 12.3 & 6.5 & 0.082 \\
\hline 764.89532 & 88.686 & 20.53 & 11.3 & 6.8 & 0.080 \\
\hline 766.87982 & 46.630 & 18.25 & 11.6 & 4.9 & 0.119 \\
\hline 767.80618 & 33.753 & 16.82 & 11.2 & 4.6 & 0.134 \\
\hline 769.77114 & 53.710 & 20.40 & 12.3 & 5.6 & 0.099 \\
\hline 770.85930 & 78.537 & 19.60 & 11.3 & 5.3 & 0.103 \\
\hline 790.70926 & 88.310 & 20.55 & 10.9 & 5.5 & 0.094 \\
\hline 797.73001 & 96.514 & 19.70 & 12.1 & 5.7 & 0.099 \\
\hline \multicolumn{6}{|l|}{ OGLE-35a } \\
\hline 759.79751 & -145.398 & 3.74 & 58.6 & 16.8 & 0.367 \\
\hline 764.89532 & -134.986 & 3.63 & 57.1 & 17.2 & 0.365 \\
\hline 766.87982 & -151.252 & 3.59 & 58.0 & 16.0 & 0.399 \\
\hline 769.77114 & -159.365 & 3.84 & 59.2 & 16.9 & 0.357 \\
\hline 770.85930 & 42.947 & 3.75 & 56.7 & 16.1 & 0.376 \\
\hline 790.70926 & 10.277 & 3.47 & 55.0 & 16.8 & 0.383 \\
\hline 797.73001 & -117.998 & 3.48 & 54.9 & 17.1 & 0.375 \\
\hline \multicolumn{6}{|l|}{ OGLE-35b } \\
\hline 759.79751 & 21.308 & 3.78 & 56.7 & 16.8 & 0.357 \\
\hline 764.89532 & 11.174 & 3.60 & 55.9 & 17.2 & 0.364 \\
\hline 766.87982 & 27.804 & 3.64 & 56.8 & 16.0 & 0.390 \\
\hline 769.77114 & 35.511 & 3.78 & 55.9 & 16.9 & 0.353 \\
\hline 770.85930 & -165.670 & 3.86 & 56.6 & 16.1 & 0.365 \\
\hline 790.70926 & -133.420 & 3.48 & 55.4 & 16.8 & 0.384 \\
\hline 797.73001 & -3.016 & 3.42 & 54.9 & 17.1 & 0.382 \\
\hline \multicolumn{6}{|l|}{ OGLE-48 } \\
\hline 766.79458 & -0.392 & 0.79 & 203.7 & 12.5 & 4.336 \\
\hline 767.72839 & -0.798 & 0.89 & 323.6 & 7.8 & 7.774 \\
\hline 768.84710 & 4.351 & 1.00 & 232.4 & 15.8 & 2.895 \\
\hline 770.82050 & 6.071 & 1.17 & 383.9 & 8.9 & 5.645 \\
\hline \multicolumn{6}{|l|}{ OGLE-49 } \\
\hline 759.72111 & -106.352 & 12.44 & 9.7 & 2.2 & 0.338 \\
\hline 764.85708 & -106.928 & 13.39 & 7.4 & 2.5 & 0.246 \\
\hline 766.79458 & -107.230 & 19.76 & 9.5 & 2.7 & 0.174 \\
\hline 767.72839 & -106.680 & 10.80 & 9.3 & 2.0 & 0.434 \\
\hline 768.84710 & -106.965 & 25.41 & 9.1 & 4.6 & 0.085 \\
\hline 769.85598 & -107.327 & 17.46 & 9.4 & 2.6 & 0.204 \\
\hline 770.82050 & -106.830 & 16.52 & 9.7 & 2.6 & 0.218 \\
\hline 797.76880 & -106.970 & 20.26 & 9.4 & 3.1 & 0.150 \\
\hline
\end{tabular}

low mass stellar companion or a background star may also affect the determination of the stellar parameters.

For the fast rotating stars in our sample $(v \sin i \geq$ $20 \mathrm{~km} \mathrm{~s}^{-1}$ ), the method described in Santos et al. (2004) is not applicable, given that the measurement of individual equivalent widths is not accurate enough because of line blending. In these cases we have adopted a simpler approach, and have determined very approximate effective temperatures for the stars by visual comparison of the combined spectra with synthetic spectra convolved with a rotational profile to take the projected rotational velocity into account.

Stellar spectroscopic parameters are given in Table 2. The lines of OGLE-TR-48 are too rotationally broadened for a spectral type estimation. Our $\log g$ and $[\mathrm{Fe} / \mathrm{H}]$ estimates are all very uncertain, they simply indicate that the target objects are dwarfs and have solar or above-solar metallicities. 
Table 1. continued.

\begin{tabular}{|c|c|c|c|c|c|}
\hline $\begin{array}{r}\text { BJD } \\
{[-2452000 \mathrm{~d}]} \\
\end{array}$ & $\begin{array}{r}R V \\
{\left[\mathrm{~km} \mathrm{~s}^{-1}\right]} \\
\end{array}$ & $\begin{array}{r}\text { depth } \\
{[\%]} \\
\end{array}$ & $\begin{array}{r}F W H M \\
{\left[\mathrm{~km} \mathrm{~s}^{-1}\right]}\end{array}$ & SNR & $\begin{array}{r}\sigma_{\mathrm{RV}} \\
{\left[\mathrm{km} \mathrm{s}^{-1}\right]} \\
\end{array}$ \\
\hline \multicolumn{6}{|l|}{ OGLE-55 } \\
\hline 759.79751 & -12.937 & 5.62 & 49.2 & 7.0 & 0.536 \\
\hline 764.89532 & 5.990 & 5.55 & 50.7 & 7.3 & 0.528 \\
\hline 766.87982 & 24.803 & 4.20 & 48.4 & 5.8 & 0.857 \\
\hline 767.80618 & 20.569 & 5.18 & 53.1 & 5.2 & 0.812 \\
\hline 769.77114 & 9.084 & 5.73 & 53.1 & 6.0 & 0.637 \\
\hline 770.85930 & 26.478 & 5.38 & 46.4 & 6.4 & 0.595 \\
\hline 790.70926 & -12.510 & 4.66 & 43.5 & 5.4 & 0.787 \\
\hline 797.73001 & -21.156 & 5.47 & 49.1 & 7.5 & 0.514 \\
\hline \multicolumn{6}{|l|}{ OGLE-56 } \\
\hline 759.79751 & -48.284 & 30.47 & 10.1 & 12.2 & 0.043 \\
\hline 764.89532 & -48.528 & 30.83 & 10.2 & 11.6 & 0.044 \\
\hline 766.87982 & -48.241 & 29.47 & 10.7 & 9.2 & 0.050 \\
\hline 767.80618 & -48.279 & 27.69 & 10.5 & 8.0 & 0.056 \\
\hline 769.77114 & -48.516 & 29.69 & 10.3 & 10.3 & 0.047 \\
\hline 770.85930 & -48.485 & 29.44 & 10.5 & 9.8 & 0.049 \\
\hline 790.70926 & -48.306 & 28.20 & 10.3 & 9.0 & 0.052 \\
\hline 797.73001 & -48.518 & 29.11 & 10.1 & 9.9 & 0.048 \\
\hline 809.76233 & -48.550 & 36.90 & 8.1 & 2.7 & 0.087 \\
\hline 810.59822 & -48.126 & 40.30 & 8.6 & 2.3 & 0.094 \\
\hline 810.83456 & -48.440 & 37.40 & 9.1 & 2.3 & 0.107 \\
\hline 811.63656 & -48.011 & 40.10 & 8.2 & 3.8 & 0.057 \\
\hline 811.83617 & -48.212 & 39.50 & 8.1 & 3.0 & 0.073 \\
\hline \multicolumn{6}{|l|}{ OGLE-58 } \\
\hline 759.75994 & 51.016 & 11.48 & 13.1 & 15.6 & 0.070 \\
\hline 764.81842 & 51.091 & 11.69 & 12.6 & 13.8 & 0.075 \\
\hline 766.84092 & 51.115 & 11.54 & 13.0 & 14.4 & 0.074 \\
\hline 767.76871 & 51.200 & 11.04 & 12.6 & 10.5 & 0.098 \\
\hline 768.76694 & 51.169 & 11.70 & 12.7 & 16.5 & 0.066 \\
\hline 769.81610 & 51.051 & 11.60 & 13.0 & 15.1 & 0.071 \\
\hline 770.90035 & 50.996 & 11.35 & 13.1 & 13.8 & 0.078 \\
\hline 791.72546 & 51.004 & 11.38 & 12.9 & 15.3 & 0.071 \\
\hline \multicolumn{6}{|l|}{ OGLE-59a } \\
\hline 759.75994 & 9.765 & 3.32 & 49.4 & 13.3 & 0.479 \\
\hline 764.81842 & 8.670 & 3.30 & 49.5 & 10.8 & 0.593 \\
\hline 766.84092 & 9.714 & 3.30 & 46.6 & 12.0 & 0.518 \\
\hline 767.76871 & 8.835 & 3.30 & 49.5 & 8.0 & 0.800 \\
\hline 768.76694 & 8.890 & 3.43 & 51.3 & 14.1 & 0.446 \\
\hline 769.81610 & 8.119 & 3.43 & 49.6 & 12.9 & 0.479 \\
\hline 770.90035 & 9.113 & 3.30 & 49.5 & 10.8 & 0.593 \\
\hline 791.72546 & 9.363 & 3.30 & 49.5 & 12.4 & 0.517 \\
\hline \multicolumn{6}{|l|}{ OGLE-59b } \\
\hline 759.75994 & 61.728 & 2.28 & 27.0 & 13.3 & 0.515 \\
\hline 764.81842 & 3.619 & 3.11 & 36.7 & 10.8 & 0.542 \\
\hline 766.84092 & -44.414 & 2.82 & 28.6 & 12.0 & 0.475 \\
\hline 767.76871 & -2.520 & 2.46 & 27.2 & 8.0 & 0.796 \\
\hline 768.76694 & 60.252 & 2.63 & 25.3 & 14.1 & 0.408 \\
\hline 769.81610 & -44.422 & 2.89 & 26.4 & 12.9 & 0.415 \\
\hline 770.90035 & 13.946 & 2.92 & 34.8 & 10.8 & 0.562 \\
\hline 791.72546 & -1.157 & 3.70 & 35.5 & 12.4 & 0.391 \\
\hline
\end{tabular}

A spectral classification of 7 of our targets (OGLE-TR-5, $6,8,10,12,19$ and 35) was previously done by Dreizler et al. (2002). Our determination of the stellar spectroscopic parameters is in agreement with their result.
Table 2. Parameters from the spectroscopic analysis. Rotation velocities $v \sin i$ are computed from the analysis of the CCF. $T_{\text {eff }}, \log g$, $[\mathrm{Fe} / \mathrm{H}]$ : temperature, gravity and metallicity are computed from the analysis of the spectral lines in case of low $v \sin i$. For high rotation, $T_{\text {eff }}$ was estimated roughly by comparison with a synthetic spectrum. It was not possible to determine the spectral types of binaries (identified with label $a$ and $b$ ) that include two moving components in the spectra.

\begin{tabular}{|c|c|c|c|c|}
\hline Name & $\begin{array}{r}v \sin i \\
{\left[\mathrm{~km} \mathrm{~s}^{-1}\right]}\end{array}$ & $\begin{array}{l}T_{\text {eff }} \\
{[\mathrm{K}]}\end{array}$ & $\log g$ & {$[\mathrm{Fe} / \mathrm{H}]$} \\
\hline 5 & $87.8 \pm 1.8$ & $6700 \pm 500$ & - & - \\
\hline 6 & $22.6 \pm 0.3$ & $5700 \pm 500$ & - & - \\
\hline 7 & $30.7 \pm 0.2$ & $6500 \pm 500$ & - & - \\
\hline $8 \mathrm{a}$ & $11.7 \pm 0.3$ & - & - & - \\
\hline $8 b$ & $11.3 \pm 0.4$ & - & - & - \\
\hline 10 & $7.7 \pm 0.1$ & $6220 \pm 140$ & $4.70 \pm 0.34$ & $0.39 \pm 0.14$ \\
\hline 12 & $7.7 \pm 0.2$ & $6440 \pm 300$ & $4.90 \pm 0.35$ & $0.30 \pm 0.26$ \\
\hline 17 & $<5$ & $5870 \pm 190$ & $4.80 \pm 0.45$ & $-0.06 \pm 0.21$ \\
\hline 18 & $44.3 \pm 0.9$ & $6500 \pm 500$ & - & - \\
\hline 19 & $6.0 \pm 0.2$ & $5250 \pm 300$ & $4.0 \pm 0.50$ & $-0.10 \pm 0.30$ \\
\hline $33 a$ & $45.3 \pm 0.5$ & $6700 \pm 500$ & - & - \\
\hline $33 b$ & $\sim 32$ & - & - & - \\
\hline 34 & $6.3 \pm 0.3$ & $6520 \pm 340$ & $4.50 \pm 0.48$ & $0.32 \pm 0.31$ \\
\hline $35 \mathrm{a}$ & $34.3 \pm 1.1$ & - & - & - \\
\hline $35 b$ & $34.8 \pm 0.8$ & - & - & - \\
\hline 48 & $>100$ & - & - & - \\
\hline 49 & $<5$ & $5000 \pm 500$ & - & - \\
\hline 55 & $30.5 \pm 0.6$ & $6000 \pm 500$ & - & - \\
\hline 56 & $<5$ & $5970 \pm 150$ & $4.20 \pm 0.38$ & $0.17 \pm 0.19$ \\
\hline 58 & $14.6 \pm 0.1$ & $6500 \pm 500$ & - & - \\
\hline $59 \mathrm{a}$ & $34.1 \pm 0.7$ & - & - & - \\
\hline $59 b$ & $20.9 \pm 1.1$ & - & - & - \\
\hline
\end{tabular}

\section{Light curve analysis}

\subsection{Existence and periodicity of a transit signal}

When the amplitude of the radial velocity variation is small, which may indicate the presence of a planet or a blend with a background eclipsing binary, one must first consider the possibility that the detected photometric signal is not a bona fide transit or eclipse, or that the period of the signal is incorrect. The OGLE photometric data are subject to systematic intranight drifts in calibration to the level of $0.01 \mathrm{mag}$, similar to the depth of the smallest detected transit signal. Given the fact that the transit candidates were detected among about 60000 light curves, some of them may be artefacts. Either the whole detection is spurious or, more likely, one of the detected transit is an artifact. If there are only two detected transits, this would imply that the periodicity of the event is unknown, and therefore that no information can be derived from the absence of radial velocity variations.

To quantify the reliability of the transit detection, we divided the value of the transit depth by its uncertainty, which yields a "confidence factor" for the existence of the transit, and plotted this factor as a function of the number of transits 


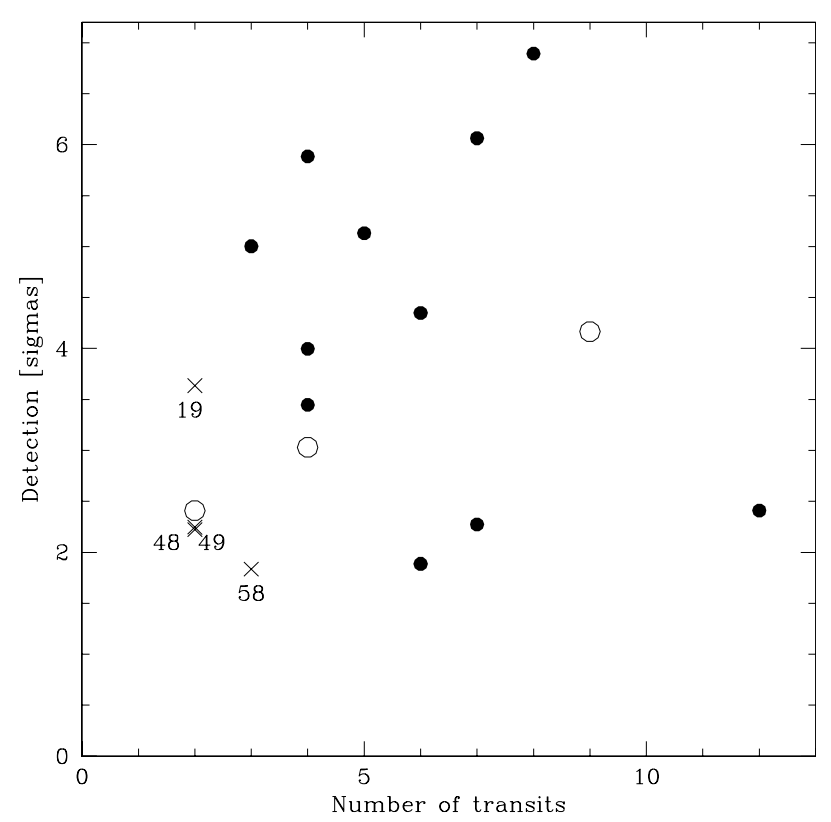

Fig. 3. Number of detected transits vs. $d / \sigma$, where $d$ is the depth of the transit signal and $\sigma$ the photometric uncertainty. Open symbols indicate objects for which the period had to be modified according to the radial velocity data (OGLE-TR-12, 17 and 59), crosses the objects for which no radial velocity variation was detected.

(see Fig. 3). Objects with only two or three detected transits and a low confidence factor are more likely to be artefacts. This is the case of objects OGLE-TR-19, 48, 49 and 58 which are possible spurious transit detections and are discussed individually below (Sect. 5.4 and Fig. 10).

When the radial velocity data did not obviously confirm the period of the transit candidates, we also studied the light curve to see if the transit signal were compatible with other periods. For instance, in a two-transit case, several divisors of the interval between the two transits can be possible to put the data in phase. For OGLE-TR-12, 17 and 59, another period than the one given by Udalski et al. (2002a, 2002b) was found to phase the light curve and radial velocity data perfectly. For equalmass double-lined eclipsing binaries (OGLE-TR-8 and 35), the correct period is twice the OGLE period because both transits and anti-transits are present in the photometric curve.

\subsection{Sinusoidal variations in the light curve}

As repeated in Sirko \& Paczynski (2003), close binaries can induce variability on the mmag level in the light curve in phase with the transit signal period. If the light of the secondary is not negligible compared to the primary, an anti-transit signal can be visible. Even in the absence of anti-transit, the ellipsoidal deformation of the primary under the gravitational influence of the secondary causes sinusoidal variations in the light curve with double the phase of the orbital period. Such sinusoidal signals were fitted to the OGLE transit candidate light curve by Sirko \& Paczinski (2003).

We have repeated their procedure and find very close results except for the objects for which the period had to be modified according to the radial velocity data (OGLE-TR-12, 17, and 59) and for the SB2 (OGLE-TR-8 and 35). Such a procedure clearly indicates that OGLE-TR-5, 8, 18 and 35 have a massive companion in the stellar mass range.

When significant, the periodic sinusoidal signals in the light curves were subtracted from the data before the analysis of the transit shape.

\subsection{Analysis of the transit shape}

The depth, width and general shape of the transit signal depend on a combination of physical variables, mainly the radius ratio $\bar{r}$, the primary radius $R$ and the impact parameter $b$ (or, equivalently for circular orbits, the angle $i$ of the normal to the orbital plane with the line-of-sight) and the orbital eccentricity. It is also weakly dependent on the total mass $(m+M)-$ via the orbital period and semi-major axis for a Keplerian orbit and the limb darkening coefficients. The parameter $\bar{r}$ is mainly constrained by the transit depth, $R \cdot(m+M)^{-1 / 3}$ by its duration, and $b$ by its shape. We assumed that all orbits were circular $(e=0)$. Low-period binaries below $P \sim 10$ days are observed to have circularized orbits (Levato 1976), and all objects in our sample have lower periods except OGLE-TR-17, which indeed shows indications of a small eccentricity in the radial velocity curve (see Sect. 5.1). In all other cases of large-amplitude variations the radial velocity residuals do not show significant variations from a circular orbit.

The light curves were fitted by non-linear least-squares fitting with analytic transit curves computed according to Mandel \& Agol (2002), using a quadratic limb darkening model with $u 1+u 2=0.3$. Notice that this is different from the coefficients used for the determination of the rotational velocity, because the wavelengths are different. The OGLE data were obtained with an $I$ filter while the spectra are centered on the visible. The fitted parameters were $\bar{r}, V_{T} / R$ and $b$, where $V_{T}$ is the transversal orbital velocity at the time of transit.

Broadly, there are two kinds of transit shape. Either the transit signal is broad and flat and $b$ has a firm upper bound - a central transit - and in that case $\bar{r}$ and $V_{T} / R$ are very well constrained by the depth and duration of the transit. Or the $V$-shaped or indefinite signal shape allows high values of $b$ - a grazing transit - and in that case $\bar{r}$ and $V_{T} / R$ are correlated with $b$ and cannot be well determined independently. In real terms, this means that the signal comes either from a small body transiting rapidly across the primary, or a larger body partially obscuring the primary in a slower grazing eclipse. For illustration, Fig. 4 shows the fits of the transits of all our candidates.

The OGLE photometric data are subject to strongly covariant noise. For many objects the systematic drift during the nights - especially near the beginning and end of the night - is of the same order as the random noise or even larger (see lowest panel on Fig. 10). Therefore, not all the data points can be considered as independent estimators on the fitted curve and an error analysis from a chi-square distribution will significantly underestimate the uncertainties.

To compute realistic uncertainties in the values of the transit parameters we used a technique based on the permutation of 


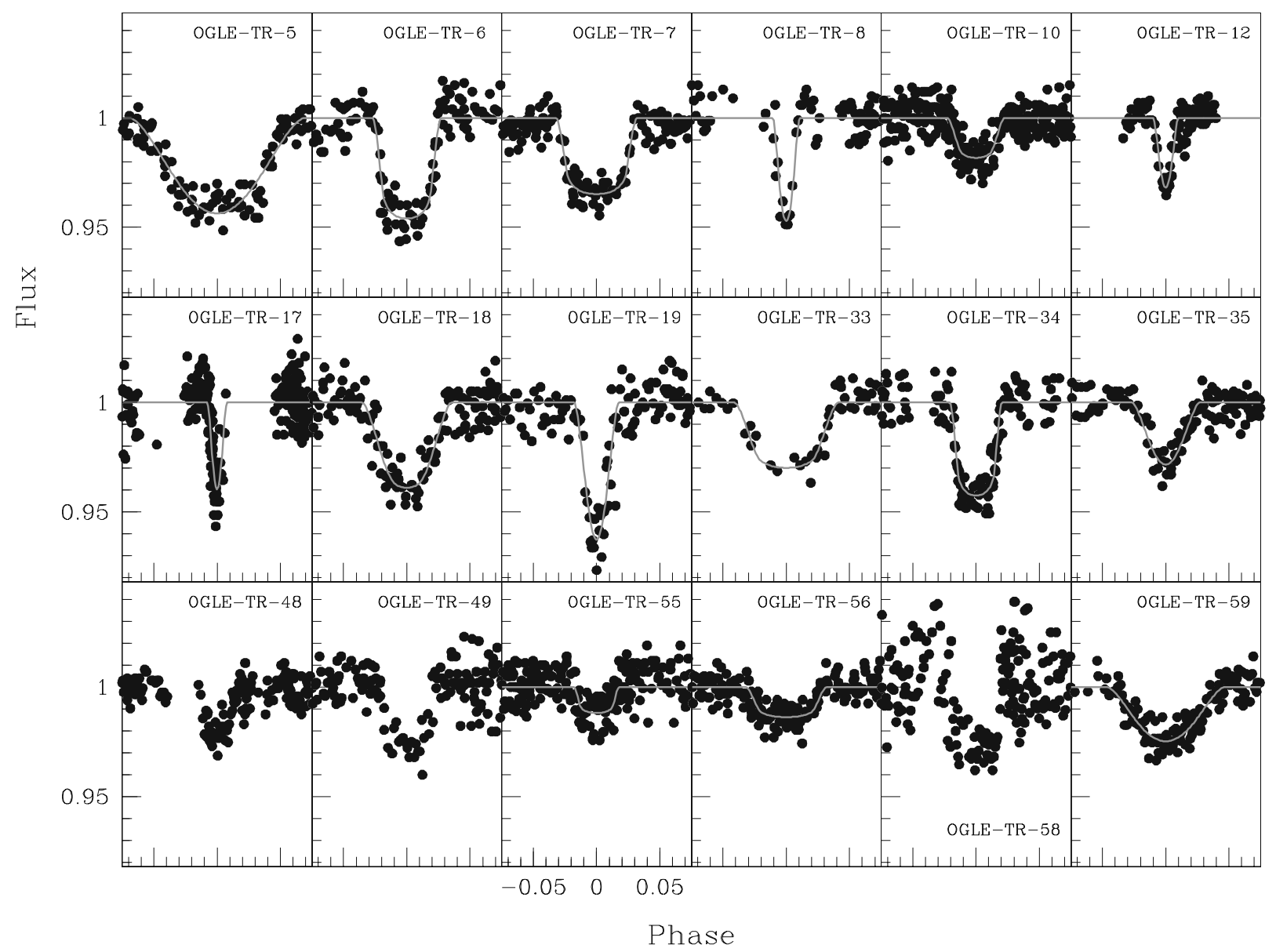

Fig. 4. Phase-folded light curve and best-fit transit curve for all the OGLE candidates followed.

the residuals. We compute the residuals by removing the bestfit solution for the transit shape, then exchange the residuals of one night with those of another randomly chosen night. The fitting procedure is then repeated for the resulting curve with the shuffled residuals, leaving the period as a free parameter. Many Monte Carlo realizations are then carried out to estimate the dispersion of the fitted parameters.

This permutation procedure has the advantage of "letting the data speak for themselves" and automatically incorporates the real characteristics of both the random noise and the systematic intra-night drifts. Its use is especially relevant for objects where the transit was covered in a low number of nights. In that case, intra-night systematic drifts can significantly alter the transit shape, and a $\chi^{2}$ analysis will yield much too low uncertainties. Figure 5 gives an example of the residual permutation procedure for OGLE-TR-12.

\subsection{Synthesis of the spectroscopic and photometric constraints}

The different constraints are combined by chi-square minimization to obtain an estimate of the physical characteristics of the two bodies involved in the transit. In most cases, constraints overlap and allow one or several coherence checks between the different lines of inquiry.
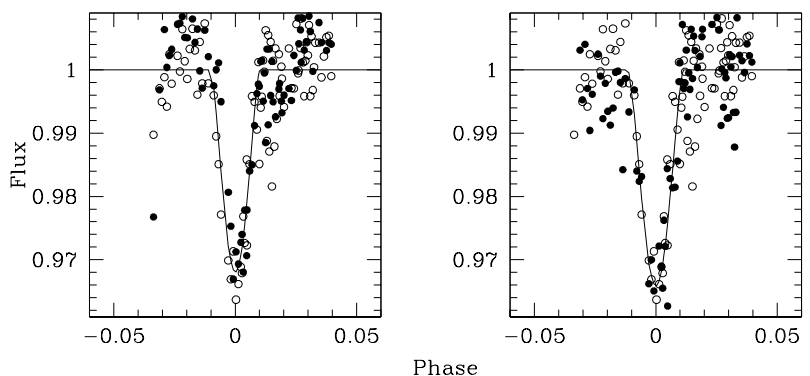

Fig. 5. Illustration (for OGLE-TR-12) of the residual permutation method for the estimation of the uncertainties. In both panels the open symbols indicate the original data, and the dots indicate two realizations (among a total of 100 realizations) of the data with residuals permutated between different nights. The lines indicate the best fits to the permitted data. The resulting $\bar{r}$ values are 0.254 and 0.292 respectively. Because the transit coverage is constituted by only two nights, systematic trends in the residuals have a large effect on the resulting parameters. In the case of OGLE-TR-12, this error estimate leads to a much higher 1-sigma interval than a $\chi^{2}$ analysis.

The measured rotation velocity $v \sin i\left(\right.$ in $\mathrm{km} \mathrm{s}^{-1}$ ) is related to the radius of the primary (in solar unit) and the period (in days) through:

$v \sin i=50.6 \cdot \frac{R}{P} \cdot \sin i$. 
For a circular orbit, the semi-amplitude of the radial velocity variation (in $\mathrm{km} \mathrm{s}^{-1}$ ) is related to the masses (in solar unit) and period (in days) through:

$K=214 \cdot \frac{m}{(m+M)^{2 / 3}} \cdot P^{-1 / 3}$.

The impact parameter $b$ and transit velocity by radius of the primary $V_{T} / \mathrm{R}$ are related to the orbital and physical parameters $R$, $m, M$ (in solar unit), $P$ (in days) and $i$, for a circular Keplerian orbit, in the following way:

$b=\frac{a \cdot \cos i}{R}$,

$V_{T} / R=2 \pi \cdot \frac{a}{P \cdot R}$

with $a$ (in solar radius) given by Kepler's third law:

$a=4.20 \cdot P^{2 / 3} \cdot(m+M)^{1 / 3}$.

The radius ratio is obviously found from to the stellar radius $R$ and companion radius $r$ :

$\bar{r}=r \cdot R^{-1}$.

Finally, the spectroscopic determination of the temperature, gravity and metallicity of the primary provides independent constraints on $R$ and $M$ via the stellar evolution models. We used the relation between physical and observable parameters provided by the models of Girardi et al. (2002).

$\left(\log T_{\text {eff }}, \log g,[\mathrm{Fe} / \mathrm{H}], R\right)=f(M$, age,$Z)$.

The constraints of Eqs. (1)-(6) were combined by chi-square minimization. In most cases, $R$ is precisely determined from the rotation velocity. Then $M$ is constrained on the one hand by the spectroscopic parameters applied by the models, on the other hand by the $V_{T} / R$ factor in the fit and the transit curve. Parameter $r$ then proceeds from $R$ and $\bar{r}$, and $m$ from $M$ and $K$. Parameter $i$ is mainly derived from $b$. A low value of the minimum $\chi^{2}$ ensures that the different constraints are coherent. This procedure yields very satisfactory accuracies on all the derived parameters. Note that the resulting $r$ and $m$ are only weakly dependent on the accuracy of the stellar evolution models, because $R$ is primarily determined from the rotation velocity and $M$ enters the determination of $m$ with a $2 / 3$ exponent.

There are two cases for which the accuracy of the final parameters is lower: 1$)$ in cases when high values of $b(b \sim 1)$ are compatible with the light curve data, there is some degeneracy between the impact parameter, the duration of the eclipse and the ratio of the radii. In that case the upper error bar on $r$ can be large because the light curve data are compatible with a grazing eclipse by a larger object. This is the case for OGLE-TR-12, 17 and 55.2) If the primary is not in synchronous rotation, then $R$ and $M$ are much more weakly constrained by Eqs. (5) and (6). In that case the adequacy of the stellar evolution models becomes more important. This is the case for OGLE-TR-34.

\section{Results}

In this section we present our results of Doppler follow-up and light curve analysis. Figures 6-9 show the radial velocity data phased with the period from Udalski et al. (2002a,b), or, in the case of OGLE-TR 8, 12, 17, 35, and 59, with the modified period obtained from our analysis. If the radial velocity variations are caused by the transiting objects, then phase $\phi=0$ must correspond to the point on the curve at the center-of-mass-velocity where the velocity is decreasing, which provides a further constraint. Results of the fit of the transit shape are summarized in Table 3. Notice that we deliberately do not provide the results for the 4 unsolved cases suspected to be false positives. For the two cases of grazing eclipsing binaries, the stellar parameters were not deduced from the light curve (except the impact parameter $b$ ) but from the spectroscopic orbits and the rotational velocities.

We distinguish 5 classes of objects, the low-mass-startransiting companions, the grazing eclipsing binaries, the triple systems, the planetary candidates, and the unsolved cases. For the majority of objects, we used the photometric ephemeris given by Udalski et al. (2002a,b) and the updated ephemeris available from the OGLE website ${ }^{2}$. We fixed the transit epoch $T 0$ and fitted the phased radial velocity with a circular orbit. In this way, we determined an updated or corrected period $P$, the velocity offset $V 0$, and the velocity semi-amplitude $K$. Each class of objects is described in the following subsection and the derived masses and radii are presented and discussed in Sect. 6.

\subsection{Low-mass-star-transiting companions}

The orbital parameters we derived for the 8 low-masstransiting-stellar companions OGLE-TR-5, 6, 7, 12, 17, 18, 34 and 55 are reported in Table 4 and Fig. 6.

OGLE-TR-5: This candidate is rotating very rapidly $(v \sin i \sim$ $88 \mathrm{~km} \mathrm{~s}^{-1}$ ), in synchronization with its very short orbital period (0.8 days). Systematic ellipsoidal variations in the light curve reveal the deformation of the primary and therefore the massive nature of the secondary. The mass obtained from the transit duration and radial velocity semiamplitude is compatible with the spectral type and radius for a $\mathrm{G}+\mathrm{M}$ binary, and all parameters can be determined precisely.

OGLE-TR-6: The synchronized rotation of this target allows a precise determination of its radius. The transit shape shows that the impact parameter is small, and therefore all parameters can be computed precisely with our "standard" procedure.

OGLE-TR-7: The synchronized rotation of this target allows a precise determination of its radius. The transit shape shows that the impact parameter is small, and therefore all parameters can be computed precisely with our "standard" procedure. The computed values of $R$ and $M$ are compatible with the spectral type of a main-sequence $F$ dwarf.

\footnotetext{
${ }^{2}$ http://bulge.princeton.edu/ ogle/ogle3/transits/ transit_news.html
} 

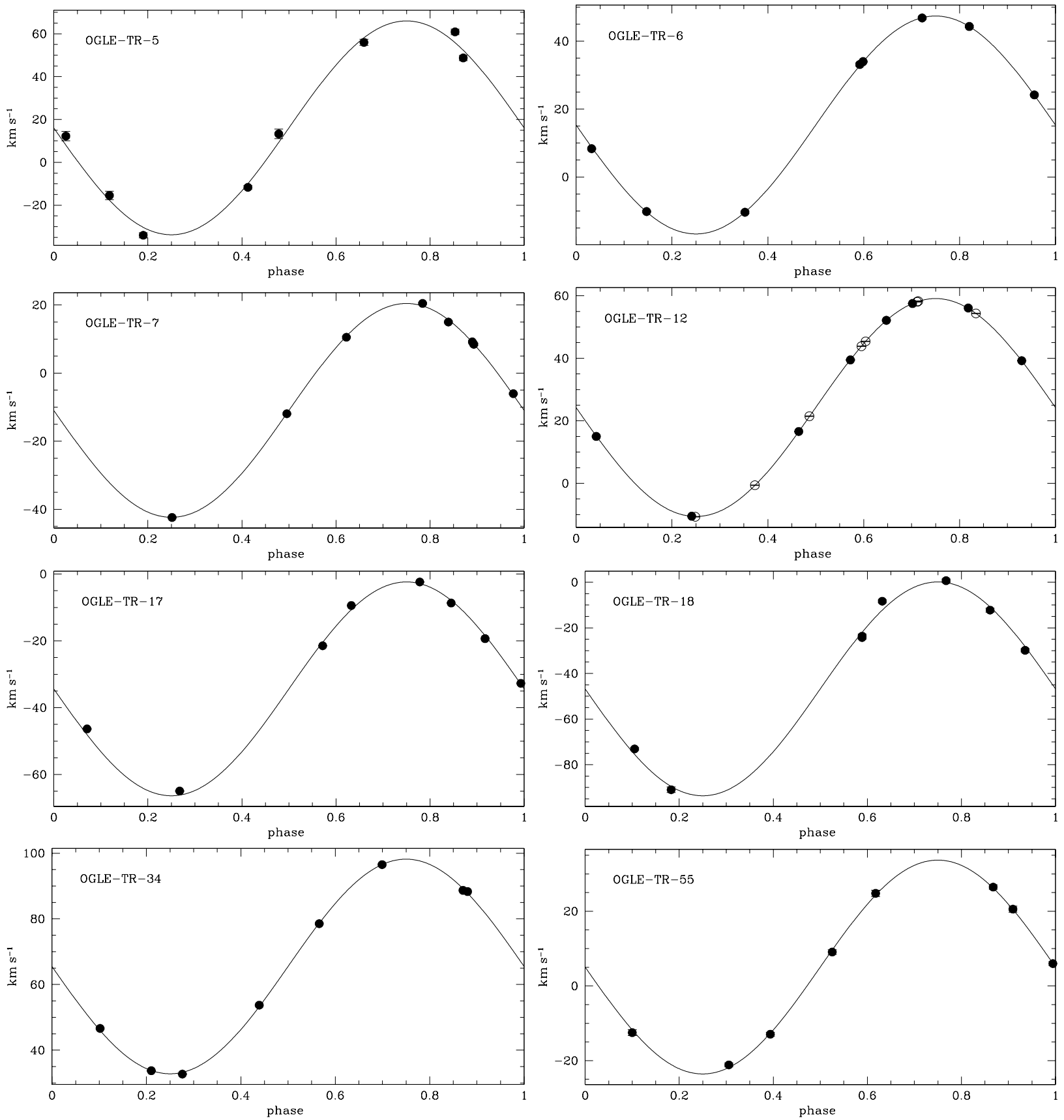

Fig. 6. Phase-folded radial velocities of the low mass star transiting companions. For OGLE-TR-12, black and white points correspond to FLAMES and UVES measurements, respectively.
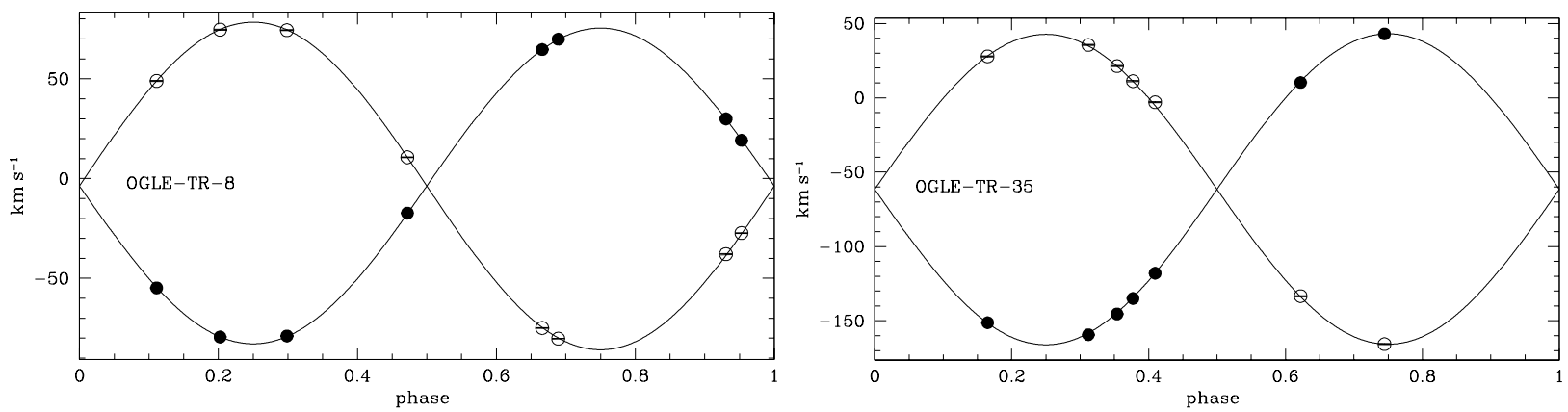

Fig. 7. Phase-folded radial velocities of grazing eclipsing binaries. Black and white points correspond to component $a$ and $b$, respectively. 

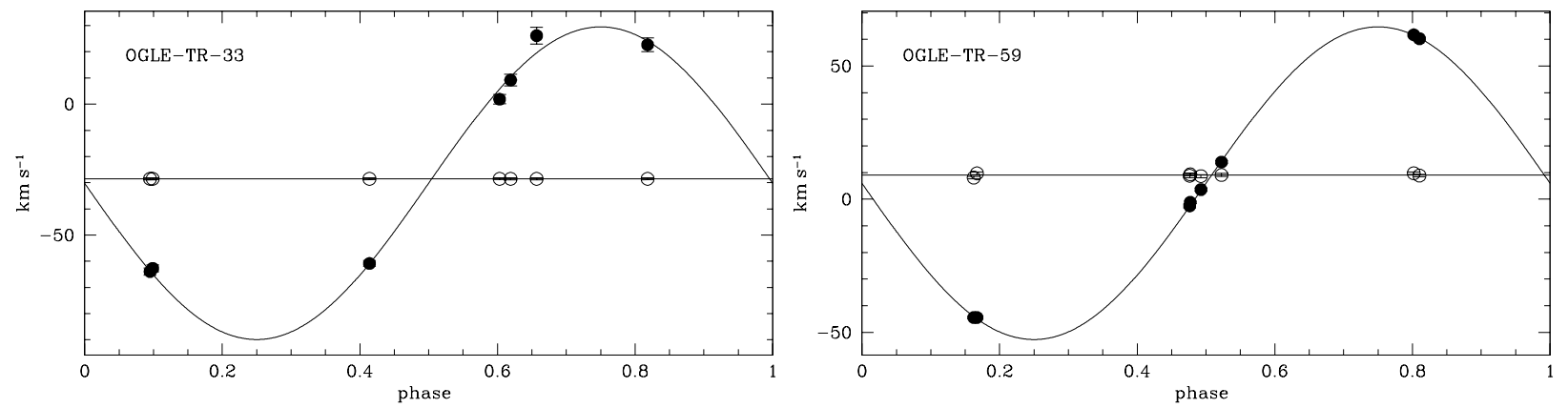

Fig. 8. Phase-folded radial velocities of triple system. Black and white points correspond respectively to component $b$ and $a$.
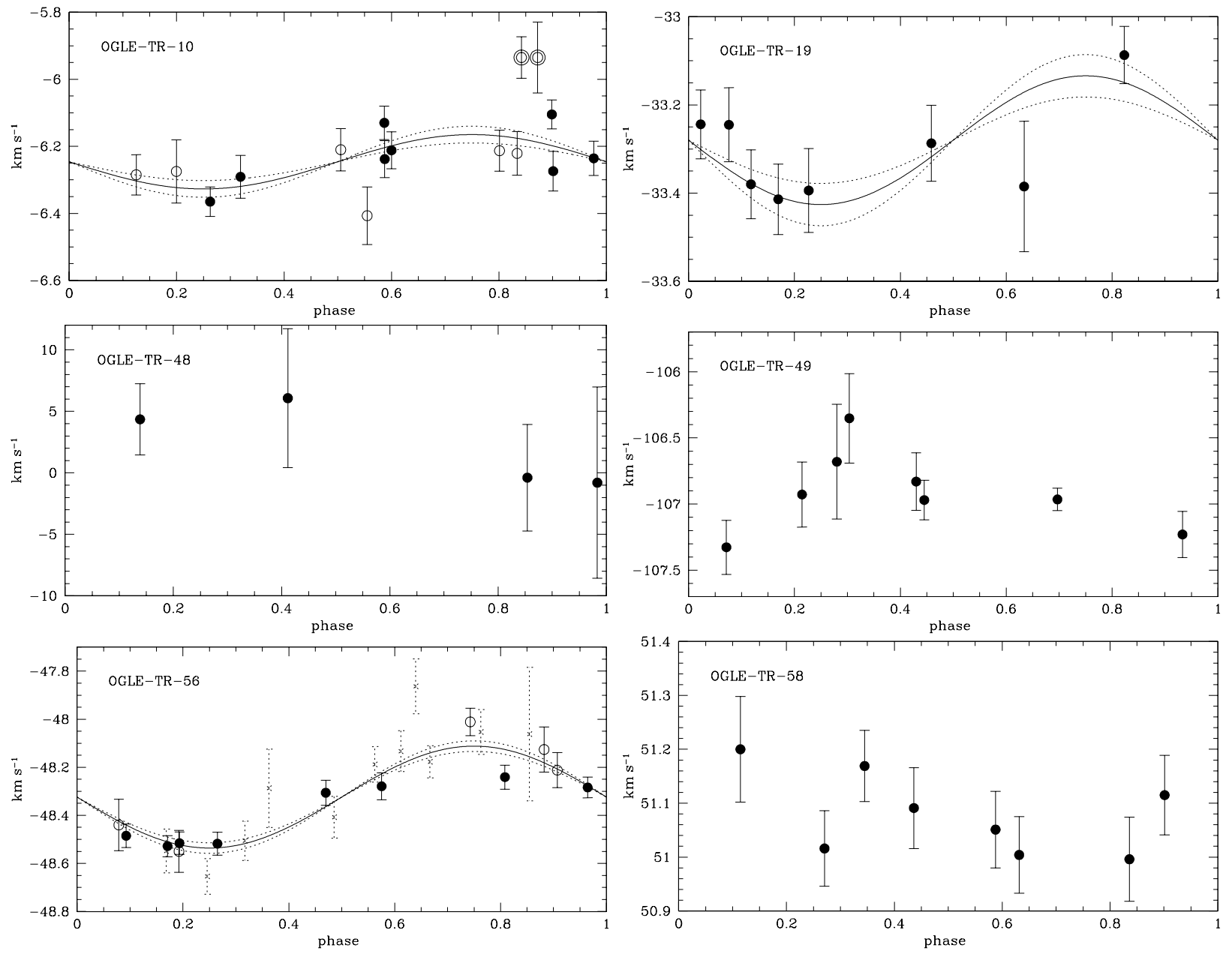

Fig. 9. Phase-folded Doppler measurements of planetary candidates and unsolved cases. For OGLE-TR-10, black and white points correspond respectively to FLAMES and UVES measurements. Encircled points correspond to measurements made with a seeing lower than 0.9 arcsec. For OGLE-TR-56, black, white and dotted cross points correspond respectively to FLAMES, HARPS and Torres et al. (2004a) measurements. For OGLE-TR-10, 19 and 56, the dotted lines correspond to fitted curves for lower and upper 1-sigma intervals of semi-amplitude $K$.

OGLE-TR-12: The radial velocity data show that this candidate is a binary system with a period 1.5 times greater than the period reported by Udalski et al. (2002a). This value is fully compatible with the light curve. The rotation is synchronized with the orbital period. The light curve indicates that the impact parameter $b$ is probably high, causing increased uncertainty in $r$ because of the degeneracy between impact parameters and the $\bar{r}$ factor. The large uncertainties are of course also due to the fact that there are only two nights in the transit. OGLE-TR-12 was observed with UVES (white points) and FLAMES (dark points). The velocity offset determined between the two set of data indicates a difference of $0.200 \mathrm{~km} \mathrm{~s}^{-1}$ between the two instruments.

OGLE-TR-17: The radial velocity data show that this target is a binary system with $P=13.8736$ days, 6 times the 
Table 3. Parameters from the transit light curve fit: $\bar{r}$ ratio of the radii of the primary and secondary bodies, $V_{T} / R$ transit velocity in units of the radius of the primary, $b$ impact parameter, $P$ revised period according to the radial velocity measurements. We deliberately do not provide the results for the 4 unsolved cases suspected to be false positives, OGLE-TR-19, 48, 49 and 58. For the two cases of SB2, stellar parameters were deduced from the spectroscopic orbits and the rotational velocities.

\begin{tabular}{rrrrrr}
\hline \hline Name & $\bar{r}$ & $\begin{array}{r}V_{T} / R \\
\left.\text { days }^{-1}\right]\end{array}$ & $b$ & {$\left[b_{\text {down }}-b_{\text {up }}\right]$} & $\begin{array}{r}P \\
\text { [days] }\end{array}$ \\
\hline 5 & $0.189 \pm 0.008$ & $22.70 \pm 1.9$ & 0.56 & {$[0.47-0.65]$} & 0.80827 \\
6 & $0.198 \pm 0.006$ & $9.76 \pm 0.44$ & 0.20 & {$[0.00-0.21]$} & 4.54881 \\
7 & $0.172 \pm 0.006$ & $13.58 \pm 0.50$ & 0.02 & {$[0.00-0.09]$} & 2.71782 \\
8 & - & - & 1.65 & {$[1.62-1.68]$} & 5.43284 \\
10 & $0.129 \pm 0.007$ & $16.80 \pm 1.22$ & 0.45 & {$[0.00-0.46]$} & 3.10140 \\
12 & $0.299[0.26-0.74]$ & $8.44 \pm 0.83$ & 1.02 & {$[0.89-1.32]$} & 8.65725 \\
17 & $0.245[0.22-0.63]$ & $8.27 \pm 0.56$ & 0.90 & {$[0.72-1.28]$} & 13.87370 \\
18 & $0.200 \pm 0.020$ & $11.78 \pm 0.83$ & 0.77 & {$[0.73-0.87]$} & 2.22803 \\
19 & - & - & - & - & - \\
33 & $0.160 \pm 0.015$ & $12.20 \pm 1.25$ & 0.68 & {$[0.59-0.77]$} & 1.95326 \\
34 & $0.188 \pm 0.002$ & $6.83 \pm 0.40$ & 0.00 & {$[0.00-0.02]$} & 8.57633 \\
35 & - & - & 1.68 & {$[1.65-1.73]$} & 2.51957 \\
48 & - & - & - & - & - \\
49 & - & - & - & - & - \\
55 & $0.109[0.107-0.150]$ & $16.45[9.21-18.72]$ & 0.60 & {$[0.00-0.85]$} & 3.18454 \\
56 & $0.114 \pm 0.004$ & $22.84[22.43-27.27]$ & 0.69 & {$[0.50-0.69]$} & 1.21192 \\
58 & - & - & - & - & - \\
59 & $0.257 \pm 0.089$ & $10.238 \pm 0.632$ & 0.99 & {$[0.82-1.31]$} & 2.99432 \\
\hline
\end{tabular}

Table 4. Orbital parameters of the low-mass-star-transiting companions, the grazing eclipsing binaries and the triple systems. For the triple systems, the component $a$ corresponds here to the contaminating third body with fixed parameters and component $b$ to the primary of an eclipsing binary with fitted parameters. ${ }^{1}$ Residuals of OGLE-TR-17 without and with eccentricity $(e=0.074) .{ }^{2}$ This value corresponds to the anti-transit epoch, the revised value is 74.65125 .

\begin{tabular}{rrrrrrr}
\hline \hline Name & $\begin{array}{r}P_{\text {OGLE }} \\
\text { [days] }\end{array}$ & $\begin{array}{r}T 0_{\text {OGLE }} \\
-2452000\end{array}$ & $\begin{array}{r}P \\
{[\text { days }]}\end{array}$ & $\begin{array}{r}K \\
{\left[\mathrm{~km} \mathrm{~s}^{-1}\right]}\end{array}$ & $\begin{array}{r}V 0 \\
{\left[\mathrm{~km} \mathrm{~s}^{-1}\right]}\end{array}$ & $\begin{array}{r}\text { O-C } \\
{\left[\mathrm{km} \mathrm{s}^{-1}\right]}\end{array}$ \\
\hline 5 & 0.8082 & 60.47118 & 0.808271 & $49.90 \pm 2.0$ & $16.1 \pm 1.4$ & 3.5 \\
6 & 4.5487 & 61.05651 & 4.54881 & $32.09 \pm 0.15$ & $15.33 \pm 0.10$ & 0.21 \\
7 & 2.7179 & 61.42566 & 2.71824 & $31.39 \pm 0.22$ & $-10.94 \pm 0.16$ & 0.37 \\
12 & 5.7721 & 61.53515 & 8.65725 & $34.862 \pm 0.025$ & $24.255 \pm 0.019$ & 0.083 \\
17 & 2.3171 & 62.35748 & 13.8737 & $31.99 \pm 0.19$ & $-34.36 \pm 0.12$ & $0.894 / 0.072^{1}$ \\
18 & 2.2280 & 61.07501 & 2.228025 & $46.87 \pm 1.48$ & $-46.75 \pm 1.03$ & 2.08 \\
34 & 8.5810 & 62.74970 & 8.57633 & $32.68 \pm 0.27$ & $65.50 \pm 0.20$ & 0.426 \\
55 & 3.18456 & 77.05049 & 3.184543 & $28.63 \pm 0.32$ & $5.06 \pm 0.19$ & 0.439 \\
\hline $8 \mathrm{a}$ & 2.7152 & 61.01604 & 5.432842 & $79.13 \pm 0.14$ & $-3.71 \pm 0.07$ & 0.260 \\
$8 \mathrm{~b}$ & 2.7152 & 61.01604 & 5.432842 & $82.10 \pm 0.15$ & $-3.71 \pm 0.07$ & 0.299 \\
$35 \mathrm{a}$ & 1.2599 & 60.98942 & 2.519569 & $104.57 \pm 0.38$ & $-61.53 \pm 0.22$ & 0.521 \\
$35 \mathrm{~b}$ & 1.2599 & 60.98942 & 2.519569 & $104.21 \pm 0.37$ & $-61.53 \pm 0.22$ & 0.611 \\
\hline 33a & 1.9533 & 60.54289 & - & 0.0 & -28.5 & - \\
$33 \mathrm{~b}$ & 1.9533 & 60.54289 & 1.95327 & $59.8 \pm 2.1$ & $-29.8 \pm 1.3$ & 3.2 \\
$59 \mathrm{a}$ & 1.49709 & 73.15416 & - & 0.0 & 9.05 & 0.495 \\
59b & 1.49709 & 73.154162 & 2.9943224 & $58.68 \pm 0.31$ & $6.01 \pm 0.22$ & 0.524 \\
\hline
\end{tabular}

value in Udalski et al. (2002a). This revision is compatible with the light curve data. The estimated rotation velocity of OGLE-TR-17 seems to indicate that this star is not synchronized which is not so surprising considering its quite large period. The shape of the transit allows high values of $b$, causing degeneracy between the parameters. Moreover, the poor phase coverage of the transit increases the uncertainties. The large $\mathrm{O}-\mathrm{C}$ residuals clearly indicate a departure from circular orbit, and a complete Keplerian fit indicates a small but significant eccentricity 


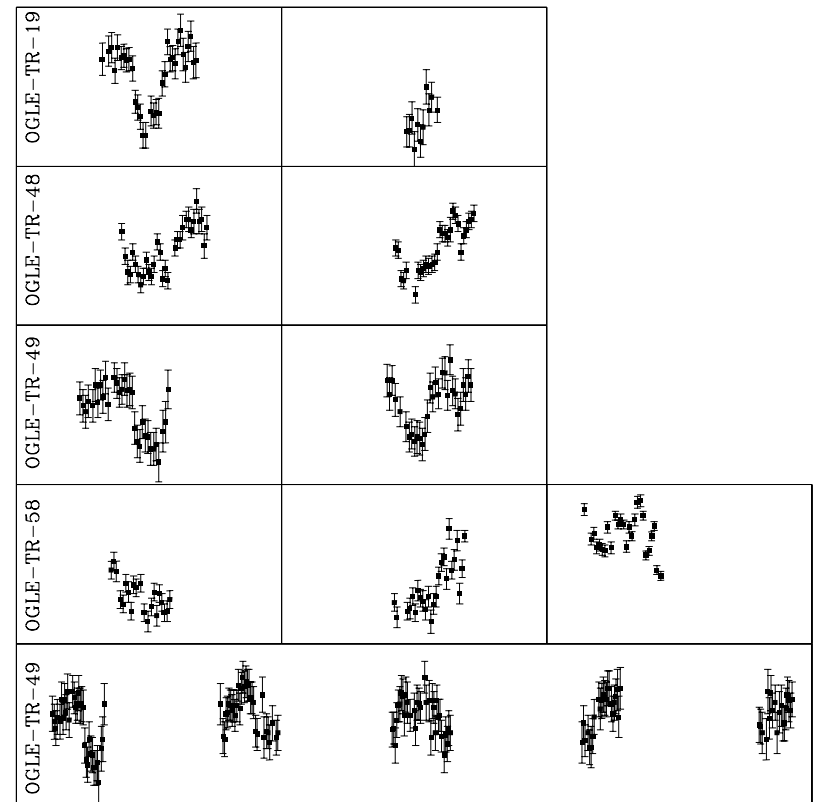

Fig. 10. Light curve data during the night of detected transits for OGLE-TR-19, 48, 49, and 58 (from top to bottom) and light curve of OGLE-TR-49 for some days after the first detected transit (bottom). The correlation of the residuals is clearly visible in the other nights as well.

of $0.074 \pm 0.008$. Note that OGLE-TR-17, with the longest period, is the only candidate that presents a significant eccentricity.

OGLE-TR-18: The rotation velocity is compatible with synchronous rotation. The fit of the transit parameters yields a high value of $b$, which increases the uncertainty in $r$.

OGLE-TR-34: The radius given by the rotation velocity assuming synchronization $\left(R=1.02 \pm 0.04 R_{\odot}\right)$ is in conflict with the spectral type $\left(R>1.25 R_{\odot}\right)$ and the light curve fit $\left(R \sim 1.9 R_{\odot}\right)$. Relaxing the assumption of synchronism appears to be the best likely solution taking into account the quite large period (8.6 days). In that case all the parameters are coherent and well determined. The radius and temperature obtained would put OGLE-TR-34 at the evolved edge of the $\mathrm{F}$ main sequence. It is possible that the radius increase at the end of the main-sequence stage has been too rapid to allow synchronization to keep pace, so that the conservation of angular momentum has slowed the rotation down to the observed value. Alternatively, the star could be too young for synchronization to have taken place yet, given the 8.6 days period.

OGLE-TR-55: The rotation velocity shows synchronization with the orbital period. The light curve, however, does not constrain the parameters with enough accuracy to confine the value of the impact parameter $b$. Because of the large line broadening caused by the high rotation velocity, our spectral type analysis yields only a rough estimate of the spectral type: F5-G8. The uncertainties in the resulting values $r$ and $m$ are correspondingly large.

\subsection{Grazing eclipsing binaries}

The orbital parameters we derived for the 2 grazing eclipsing binaries OGLE-TR- 8 and 35 are reported in Table 4 and Fig. 7.

OGLE-TR-8: The cross-correlation function of the spectra shows two components of approximately equal intensity, varying along an SB2 orbit of twice the period given by Udalski et al. (2002a), which reveals that both the eclipse and anti-eclipse of comparable magnitude were visible in the light curve. The rotation velocities show synchronous rotation for both components. The $M \sin i$ of both components are computed from the radial velocity curve, the $R \sin i$ from the rotation velocity and period, and the angle $i$ from the light curve. The eclipse has to be grazing because the components are of similar size and the depth of the eclipse is only a few percent. This constraint alone fixes $\sin i$ within a very small interval. All parameters are therefore determined with very high accuracy, showing that OGLE-TR-8 is an G-G binary, consisting of two components, both of spectral type $\mathrm{G}$.

OGLE-TR-35: The spectroscopic data show that this target is a double-line binary with a period double that given by Udalski et al. (2002a). Therefore, both the eclipse and antieclipse are visible in the light curve. As for OGLE-TR-8, the combination of the radial velocity orbits, the two rotation velocity and the transit depth fix all the parameters very precisely. OGLE-TR-35 is an F-F binary.

\subsection{Low-mass-star-transiting companions in triple systems}

The orbital parameters we derived for the 2 triple systems OGLE-TR-33 and 59 are reported in Table 4 and Fig. 8. To determine the characteristic of the second component, we subtracted in the $\mathrm{CCF}$ a fixed Gaussian or a fixed rotational profile.

OGLE-TR-33: As discussed by Konacki et al. (2003b), this candidate presents a clear blend effect visible in the line bisector as well as in the asymmetry of the bottom of the CCF. This object consists of a triple system, i.e. an eclipsing binary and a contaminating third body. In order to characterize the second spectral component, we subtracted a constant rotational profile from the CCF. The second component seems to have a rotation velocity synchronized with the orbital period and is in phase with the Udalski et al. (2002a) period. The amplitude of the radial velocity variations indicates a low-mass-star-transiting companion. A detailed analysis of this system, in full agreement with our result, have been made recently by Torres et al. (2004b).

OGLE-TR-59: This target has a double-line spectrum. However, only one set of lines shows large radial velocity variations and presents a period which is double that given by Udalski et al. (2002b). The transit epoch has to be shifted by half a revised period. Therefore, this object consists of a triple system, with an eclipsing binary and a contaminating third body, either the component of a physical triple system, or an unrelated background/foreground star. 
Table 5. Orbital parameters of planetary candidates, unsolved cases and false positives.

\begin{tabular}{rrrrrr}
\hline \hline Name & $\begin{array}{r}P_{\text {OGLE }} \\
{[\text { days }]}\end{array}$ & $\begin{array}{r}T 0_{\text {OGLE }} \\
-2452000\end{array}$ & $\begin{array}{r}K \\
{\left[\mathrm{~km} \mathrm{~s}^{-1}\right]}\end{array}$ & $\begin{array}{r}V 0 \\
{\left[\mathrm{~km} \mathrm{~s}^{-1}\right]}\end{array}$ & $\begin{array}{r}\text { O-C } \\
{\left[\mathrm{km} \mathrm{s}^{-1}\right]}\end{array}$ \\
\hline 10 & 3.1014 & 70.2190 & $0.081 \pm 0.025$ & $-6.246 \pm 0.017$ & 0.068 \\
19 & 5.2821 & 61.89798 & $0.146 \pm 0.048$ & $-33.280 \pm 0.033$ & 0.074 \\
48 & 7.2255 & 74.19693 & - & $2.30 \pm 1.45$ & 2.9 \\
49 & 2.69042 & 75.53363 & - & $-106.90 \pm 0.10$ & 0.286 \\
56 & 1.2119189 & 75.1046 & $0.212 \pm 0.022$ & $-48.324 \pm 0.018$ & 0.051 \\
58 & 4.34244 & 76.81982 & - & $51.08 \pm 0.025$ & 0.072 \\
\hline
\end{tabular}

The fixed component represents $\sim 70$ percent of the CCF surface, and the eclipsed object 30 percent. If the eclipsed object is in synchronous rotation, its rotation velocity implies $R=0.624 \pm 0.033 R_{\odot}$. The transit curve is clearly $\mathrm{V}$-shaped, indicating a grazing eclipse, so that the radius ratio of the eclipsed component to the eclipsing body is not strongly constrained. If that component obeys the $M \sim R$ relation of $\mathrm{M}$ dwarfs, then the amplitude of the radial velocity variations implies $m \sim 0.30 M_{\odot}$. Therefore the system would consist of an M-M binary with a F/G contaminant in the background. With the present data it is difficult to entirely exclude other scenarios.

\subsection{Planetary transits, unsolved cases and false positives}

Six of our targets show radial velocity variations lower than $1 \mathrm{~km} \mathrm{~s}^{-1}$, or comparable with the error bars, indicating the possibility that the transit signal is caused by a planet-mass companion. Of these, however, only one exhibits the signature of a clear orbital motion - the known planetary system OGLE-TR-56 (Konacki et al. 2003a; Torres et al. 2004a). For OGLE-TR-10, a planetary explanation is proposed. For 3 other targets, our data do not allow us to reach a conclusion but we strongly suspect a false positive transit detection as already examined in Sect. 4.1. For OGLE-TR-58 we present strong evidence of a false positive transit detection.

The orbital parameters we derived for the 6 candidates harboring low radial velocity variations OGLE-TR-10, 19, 48, 49, 56 and 58 are reported in Table 5 and Fig. 9. For these candidates we fixed the period given and updated by the OGLE team.

OGLE-TR-10: This candidate was observed with UVES (white points) and FLAMES (black points). The UVES radial velocity was corrected for the offset velocity of $0.200 \mathrm{~km} \mathrm{~s}^{-1}$ as determined on OGLE-TR-12. Note that the two higher values of UVES were made under "unfavorable" seeing conditions (seeing lower than 0.9 arcsec) and are not taken into account afterward. The fit indicates an orbital signal with $K=81 \pm 25 \mathrm{~m} \mathrm{~s}^{-1}$ which corresponds to a $0.66 \pm 0.21$ Jupiter mass companion. The reduced $\chi^{2}$ is 2.3 for a constant velocity curve and 1.4 for a circular orbit. The analysis of the transit shape and the spectroscopic parameters leads to $r=1.54 \pm 0.12 R_{\text {Jup }}$. Such a planet, if confirmed, would have a density lower than HD 209458. Also note that OGLE-TR-10 presents an excess of metallicity; it is well known that the planet-hosts discovered using radial-velocity techniques are, on average, significantly more metal-rich than average field dwarfs (e.g. Santos et al. 2001). In order to examine the possibility that the radial velocity variation is due to a blend scenario, we computed the CCF bisectors as described by Santos et al. (2002). We did not find any significant bisector effect in the CCF. We also checked the influence of the cross-correlation mask used in the CCF computation and did not find any effect. However, further observations are needed to confirm our hypothesis and a blend scenario could not be ruled out completely considering the low SNR of our data. Both our observations and the possibility of a blend scenario are in agreement with Konacki et al. (2003b).

OGLE-TR-19: The phase-folded Doppler measurements indicate a significant variation. The reduced $\chi^{2}$ is 2.3 for a constant velocity curve and 0.7 for a circular orbit. The fit gives an orbit with $K=146 \pm 48 \mathrm{~m} \mathrm{~s}^{-1}$ which corresponds to a $1.2 \pm 0.4$ Jupiter mass companion. The rotation velocity is comparable with the instrumental broadening, so that synchronous rotation is excluded. The analysis of the transit shape gives $\bar{r}=0.343$ and $b$ in the range [0.79-1.09]. The spectroscopic parameters indicate $R=1.46 \pm 0.16 R_{\odot}$ and $M=0.92 \pm 0.05 M_{\odot}$, implying $r=4.9 \pm 1.1 R_{\text {Jup }}$. Such a radius is much larger than any planet model would predict, and reinforces the odds against the planetary explanation. A single star blended with a background eclipsing binary would probably explain the data more convincingly. Our bisector analysis, limited by the high uncertainties, cannot exclude such a scenario. Alternatively, we note that only two transits were observed for this candidate (see Figs. 3 and 10), which puts OGLE-TR-19 among the less secure transit candidates. A "false positive" transit detection could not be excluded and only additional photometric and Doppler measurements will allow a definitive conclusion.

OGLE-TR-48: The spectroscopic data reveal that this target is a very rapid rotator, with $V_{\text {rot }}>100 \mathrm{~km} \mathrm{~s}^{-1}$. Consequently, only very approximate radial velocities could be computed from the spectra on only half of the spectra. These measurements do not show significant variations within the $\sim 10 \mathrm{~km} \mathrm{~s}^{-1}$ accuracy. The rotation is not synchronous with the transit period for any reasonable value of the radius of the primary. We suspect that this very high rotation 
Table 6. Summary table with the physical parameters $r, R, m$ and $M$, the orbital angle $i$ and the identification of the system.

\begin{tabular}{|c|c|c|c|c|c|c|}
\hline Name & $\begin{array}{r}r \\
{\left[R_{\odot}\right]} \\
\end{array}$ & $\begin{array}{r}R \\
{\left[R_{\odot}\right]} \\
\end{array}$ & $\begin{array}{r}m \\
{\left[M_{\odot}\right]} \\
\end{array}$ & $\begin{array}{r}M \\
{\left[M_{\odot}\right]} \\
\end{array}$ & $i$ & Comments \\
\hline 5 & $0.263 \pm 0.012$ & $1.39 \pm 0.026$ & $0.271 \pm 0.035$ & $1.12 \pm 0.21$ & $81-90$ & G-M binary \\
\hline 6 & $0.393 \pm 0.018$ & $1.99 \pm 0.068$ & $0.359 \pm 0.025$ & $1.37 \pm 0.14$ & $88-90$ & F-M binary \\
\hline 7 & $0.282 \pm 0.013$ & $1.64 \pm 0.052$ & $0.281 \pm 0.029$ & $1.32 \pm 0.21$ & $86-90$ & F-M binary \\
\hline 8 & $1.217 \pm 0.035$ & $1.27 \pm 0.035$ & $1.160 \pm 0.010$ & $1.20 \pm 0.010$ & 86 & G-G SB2 \\
\hline 10 & $0.156 \pm 0.012$ & $1.21 \pm 0.066$ & $0.00063 \pm 0.00020$ & $1.22 \pm 0.045$ & $87-90$ & possible planet \\
\hline 12 & 0.440 [0.380-1.089] & $1.47 \pm 0.065$ & $0.492 \pm 0.026$ & $1.28 \pm 0.10$ & 85 & F-M binary \\
\hline 17 & $0.371[0.325-0.956]$ & $1.52 \pm 0.110$ & $0.482 \pm 0.040$ & $1.06 \pm 0.13$ & $86-88$ & F-M binary \\
\hline 18 & $0.390 \pm 0.040$ & $1.95 \pm 0.042$ & $0.387 \pm 0.049$ & $1.18 \pm 0.22$ & $79-86$ & F-M binary \\
\hline 19 & - & - & - & - & - & unsolved case \\
\hline 33 & - & - & - & - & - & blend \\
\hline 34 & $0.435 \pm 0.033$ & $2.31 \pm 0.174$ & $0.509 \pm 0.038$ & $1.56 \pm 0.17$ & $86-90$ & F-M binary \\
\hline 35 & $1.74 \pm 0.039$ & $1.71 \pm 0.054$ & $1.200 \pm 0.009$ & $1.19 \pm 0.009$ & $80-81$ & F-F SB2 \\
\hline 48 & - & - & - & - & - & unsolved case \\
\hline 49 & - & - & - & - & - & unsolved case \\
\hline 55 & 0.209 [0.204-0.989] & $1.92 \pm 0.036$ & $0.276 \pm 0.038$ & $1.36 \pm 0.28$ & $81-90$ & F-M binary \\
\hline 56 & $0.128 \pm 0.009$ & $1.12 \pm 0.069$ & $0.00113 \pm 0.00013$ & $1.10 \pm 0.078$ & $81-83$ & confirmed planet \\
\hline 58 & - & - & - & - & - & false positive \\
\hline 59 & - & - & - & - & - & blend \\
\hline
\end{tabular}

indicates an A or early $\mathrm{F}$ star, and considering the ratio of the radii of about 0.14 , it seems unlikely that the transit could be due to a planetary companion. The upper limit on the radial velocity variations excludes a companion with mass larger than $\sim 0.15 M_{\odot}$. However, because only two partial transits were observed in the light curve (see Fig. 3 and Fig. 10), it is not possible at this stage to be confident in the validity of the transit period. The most likely explanation is that one or both detected transits are spurious.

OGLE-TR-49: No velocity variation in phase with the transit period and no synchronous rotation are observed for this candidate. The reduced $\chi^{2}$ is 1.6 for a constant velocity curve. Only two transits were observed in the light curve (see Fig. 3), which puts OGLE-TR-49 among the less secure transit candidates. The interval between the two transits is $\Delta T=21.52$ days ( 8 times the period proposed by Udalski et al. 2002b). If we adopt this largest period, the radial velocity gives $K<200 \mathrm{~m} \mathrm{~s}^{-1}$. The transit is poorly defined and the uncertainties in the transit parameters are large. The spectroscopic parameters do not allow a strong constraint on the mass and radius of the primary. Our data can unfortunately not exclude a planetary companion nor an explanation in terms of a background eclipsing binary. However, inspection of the light curve (see Fig. 10) favors an explanation in terms of a "false positive" transit detection.

OGLE-TR-56: This candidate was observed with FLAMES (black points) and the HARPS spectrograph (white points). The phase-folded Doppler measurements indicate a clear variation. The reduced $\chi^{2}$ is 9.1 for a constant velocity curve and 1.1 for a circular orbit. The fit gives an orbit with $K=212 \pm 22 \mathrm{~m} \mathrm{~s}^{-1}$ which correspond to a $1.18 \pm 0.13$ Jupiter mass companion. The analysis of the transit shape and the spectroscopic parameters lead to $r=$ $1.25 \pm 0.09 R_{\text {Jup }}$. We do not find any bisector effect in the CCF nor any influence of the cross-correlation mask used. Our characterization of OGLE-TR-56b is in agreement (within the error bars) with the revised value given by Torres et al. (2004a) and clearly confirm the planetary nature of this object.

OGLE-TR-58: This target shows no radial velocity variation and no synchronous rotation. The reduced $\chi^{2}$ is 1.0 for a constant velocity curve. The suspicion of a spurious transit signal in the light curve is even stronger than for the previous objects, because the signal detection confidence is the lowest of the whole Udalski et al. (2002a,b) sample, and the light curve shows obvious signs of variability on the level of $0.01 \mathrm{mag}$. As already noted by Konacki et al. (2003b) and Sirko \& Paczynski (2003), the mean level has increased by $0.02 \mathrm{mag}$ between one season and the next. Only two possible transits were detected, one in a night showing a clear increase of flux before the transit - so that the average flux over the night is not lower than the normal level for OGLE-TR-58 - and the other consisting of a whole night at lower flux, not a very constraining transit signal (see Fig. 10). We therefore conclude that OGLE-TR-58 is a "false positive" of the transit detection algorithm.

\section{Discussion and conclusion}

Table 6 summarizes all the available information derived for our objects following our procedure described in Sect. 4.4. The variety of cases encountered in our sample of eighteen objects is striking and covers a large part of the bestiary of possible contaminations in the search for planetary transits. 


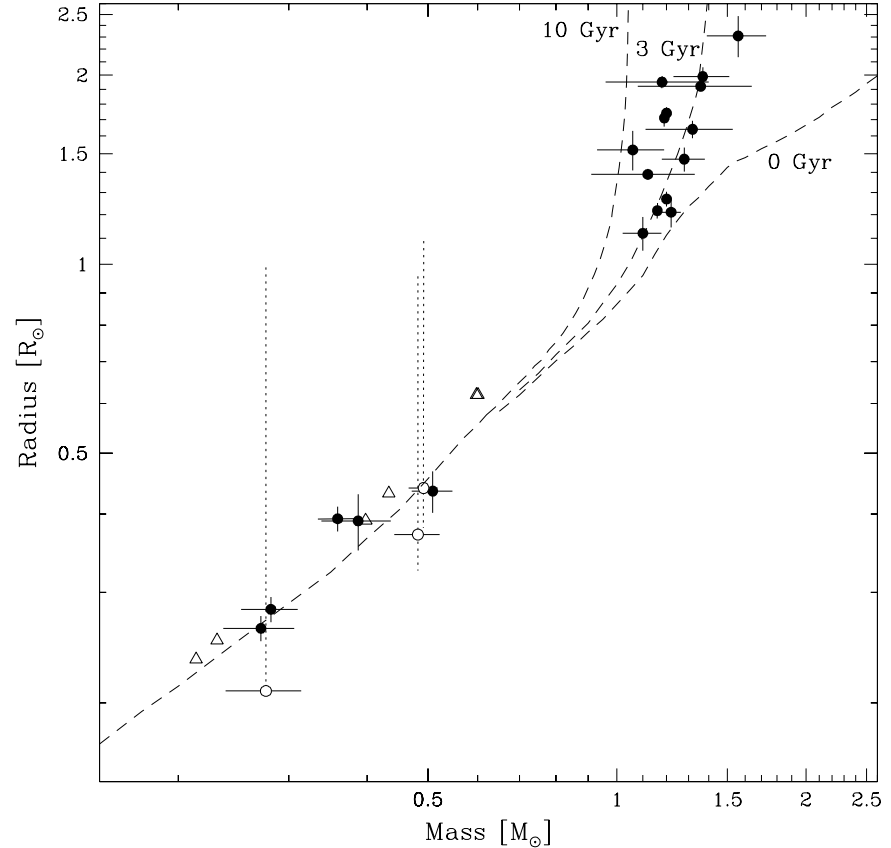

Fig. 11. The mass-radius relation for all stellar objects in our sample, primary and secondary. Open circles correspond to candidates OGLE-TR-12, 17 and 55 with a wide range of possible radii. Triangles correspond to the three known M-type eclipsing binaries. The dashed curve up to $0.6 M_{\odot}$ corresponds to the $1 \mathrm{Gyr}$ theoretical isochrones of Baraffe et al. (1998). Above $0.6 M_{\odot}$ the 3 dashed curves show the Padova model mass-radius relations for solar metallicity for three age values.

Targets OGLE-TR-5, 6, 7, 12, 17, 18, 34, and 55 have clear resolved orbits of eclipsing binaries with a large $\mathrm{F} / \mathrm{G}$ primary and a small $\mathrm{M}$ transiting companion. OGLE-TR- 8 and 35 have clear resolved orbits of equal-mass, grazing eclipsing binaries. OGLE-TR-33 and 59 have resolved orbits of eclipsing binaries in a hierarchical triple system. OGLE-TR-56 shows small radial velocity variations in agreement with Torres et al. (2004a) which confirm the planetary nature of the transiting companion. OGLE-TR-10 shows small radial velocity variations which could be due to planetary companion. OGLE-TR-19, 48 and 49 are unfortunately not yet solved but we strongly suspect false positive transit detections. OGLE-TR-58 shows no radial velocity variations and its light curve presents clear indication that the detected photometric signal is not a bona fide transit. Note that in some cases (OGLE-TR-12, 17 and 59), the initial period identified from the light curve was not correct and that in many cases our radius of the secondary is very different from the initial value of Udalski et al. (2002a,b).

Our study has yielded precise radii and masses for a certain number of low mass star companions. The mass-radius relation for these objects is given in Fig. 11. We note that no brown dwarfs were detected in our sample in agreement with the so-called brown dwarf desert for the short period companions. No stellar companions were detected in the mass domain $0.6-1.0 M_{\odot}$ because we selected in priority the smallest candidates of the OGLE survey. OGLE-TR12, 17 and 55 (white points) need additional photometric measurements in order to properly constrain the impact parameter $b$ of the transits. For the other 5 low-mass-star-transiting companions, the precision in the radius and mass determination is in the range $4.5-10 \%$ and $7-13 \%$, respectively. This precision is not at the level needed to provide a crucial test of stellar physics (e.g., Andersen 1991). However the empirical mass-radius relation remains poorly constrained because of the lack of observations of M-type eclipsing binaries. These 5 new candidates significantly increase the number of known M-type eclipsing binaries and put new observational constraints on models. For comparison we added in Fig. 11 the three known M-type eclipsing binaries (Metcalfe et al. 1996; Torres \& Ribas 2002; Ribas 2003). Although characterized by a significantly lower accuracy, our 5 low mass stars seem to follow the same departure from the models.

The OGLE fields are very crowded, and some of the targets are expected to be contaminated by background stars (or foreground fainter stars). Extrapolating the density of bright stars to fainter magnitudes indicates that there may be on average 1.4 contaminants stars per object down to $\Delta \mathrm{mag}=6$. A bright contaminant would be detected by the spectroscopy, but faint contaminants can go unnoticed and contribute a few percent to the light curve. This makes the photometric transit depth shallower, leading to an underestimation of $\bar{r}$. Note that this effect is seeing-dependent.

The effect of different assumptions for limb darkening on the derivation of the parameters from the light curve were verified using OGLE-TR-6 (central transit) and OGLE-TR-55 (grazing eclipse). Changing $\mathrm{u} 1+\mathrm{u} 2$ by 0.2 leads to a difference of the order of $2 \%$ in $\bar{r}$ and in $V_{T} / R$, while removing the limb darkening entirely changes $\bar{r}$ and $V_{T} / R$ by $\sim 8 \%$. We also test the effect of changing the limb darkening law in the derivation of the rotation velocity from the CCF. Using coefficients $u 1+u 2=0.3$ instead of $u 1+u 2=0.6$ modifies $V_{\text {rot }} \sin i$ by about $3 \%$.

This program illustrates the capability of ground-based spectrographs like FLAMES, UVES and HARPS to follow the faint transiting candidates found by photometric surveys. It demonstrates the usefulness of such a Doppler follow-up for discriminating among a large sample of possible contaminations in the search for planetary systems. We used in average only $2.5 \mathrm{~h}$ of observing time per object, thanks essentially to the very high efficiency of the FLAMES multi-fiber facility. Our analysis shows that a large part of the transiting candidates could be rejected even a priori through a fine-tuned light curve analysis (confidence factor, sinusoidal variations and transit shape). It is also clear that more accurate measurements in both photometry and radial velocity will be very useful to provide stronger constraints on the mass and radius of transiting companions, especially for the suspected planetary system OGLETR-10, for the unsolved cases OGLE-TR-19 and 49 and for the unconstrained radius of the companions of OGLE-TR-12, 17 and 55.

Acknowledgements. We are grateful to J. Smoker for support on FLAMES at Paranal. F.P. gratefully acknowledges the support of CNRS through the fellowship program of CNRS. F.B. acknowledges P. Le Strat for continuous support and advices. Support from Fundação 
para a Ciência e Tecnologia (Portugal) to N.C.S. in the form of a scholarship is gratefully acknowledged.

\section{References}

Andersen, J. 1991, A\&AR, 3, 91

Baraffe, I., Chabrier, G., Allard, F., \& Hauschilt, P. H. 1998, A\&A, 337, 403

Barban, C., Goupil, M. J., Van’t Veer-Menneret, C., et al. 2003, A\&A, 405, 1095

Bouchy, F., Pont, F., Santos, N. C., et al. 2004, A\&A, 421, L13

Brown, T. M., Charbonneau, D., Gilliland, R., et al. 2001, ApJ, 552, 699

Charbonneau, D., Brown, T. M., Latham, D., \& Mayor, M. 2000, ApJ, 529, L45

Dreizler, S., Rauch, T., Hauschildt, P., et al. 2002, A\&A, 391, L17

Dreizler, S., Hauschildt, P. H., Kley, W., et al. 2003, A\&A, 402, 791

Eggenberger, A., Udry S., \& Mayor, M. 2004, A\&A, 417, 353

Girardi, M., Manzato, P., Mezzetti, M., et al. 2002, ApJ, 569, 720

Goldreich, P., \& Tremaine, S. 1980, ApJ, 241, 425

Guillot, T. 1999, Science, 286, 72

Henry, G., Marcy, G., Butler, R., \& Vogt, S. 2000, ApJ, 529, L41

Horne, K. 2003, ASP Conf. Ser., 294, 361

Hut, P. 1981, A\&A, 99, 126

Konacki, M., Torres, G., Jha, S., et al. 2003a, Nature, 421, 507

Konacki, M., Torres, G., Sasselov, D., et al. 2003b, ApJ, 597, 1076

Kurucz, R. 1993, ATLAS9 Stellar Atmospheres Programs and $2 \mathrm{~km} \mathrm{~s}^{-1}$ Grid, CD-ROM No. 13 (Cambridge: Smithsonian Astrophys. Obs.)

Levato, H. 1976, ApJ, 203, 680
Lin, D. N. C., Bodenheimer, P., \& Richardson, D. C. 1996, Nature, 380,606

Mandel, K., \& Agol, E. 2002, ApJ, 580, 171

Mayor, M., Pepe, F., Queloz, D., et al. 2003, The Messenger, 114, 20

Mazeh, T., Naef, D., Torres, G., et al. 2000, ApJ, 532, L55

Melo, C. H. F., Covino, E., Alcala, J. M., \& Torres, G. 2001, A\&A, 378,898

Metcalfe, T. S., Mathieu, R. D., Latham, D. W., \& Torres, G. 1996, ApJ, 456, 356

Moutou, C., Pont, F., Bouchy, F., \& Mayor, M. 2004, A\&A, 424, L31

Pont, F., Bouchy, F., Queloz, D., et al. 2004, A\&A, 426, L15

Ribas, I. 2003, A\&A, 398, 239

Santos, N. C., Israelian, G., \& Mayor, M. 2001, A\&A, 373, 1019

Santos, N. C., Mayor, M., Naef, D., et al. 2002, A\&A, 392, 215

Santos, N. C., Mayor, M., Udry, S., et al. 2003, A\&A, 398, 363

Santos, N. C., Israelian, G., \& Mayor, M. 2004, A\&A, 415, 1153

Sneden, C. 1973, Ph.D. Thesis, University of Texas

Sirko, E., \& Paczynski, B. 2003, ApJ, 592, 1217

Torres, G., \& Ribas, I. 2002, ApJ, 567, 1140

Torres, G., Konacki, M., Sasselov, D., et al. 2004a, ApJ, 609, 1071

Torres, G., Konacki, M., Sasselov, D., et al. 2004b, ApJ, 614, 979

Udalski, A., Paczynski, B., Zebrun, K., et al. 2002a, Acta Astron., 52,1

Udalski, A., Zebrun, K., Szymanski, M., et al. 2002b, Acta Astron., 52,115

Udalski, A., Szewczyk, O., Zebrun, K., et al. 2002c, Acta Astron., 52, 317

Udalski, A., Pietrzynski, G., et al. 2003, Acta Astron., 53, 133

Udry, S., Mayor, M., \& Santos, N. C. 2003, A\&A, 407, 369 